\title{
Entre Mochica et Lambayeque : retracer les identités de la période Transitionnelle dans les empreintes du quotidien
}

Between Mochica and Lambayeque: retracing the identities of the Transitional period through everyday life imprints

Entre Mochica y Lambayeque: rastreando las identidades del período Transicional en las huellas del cotidiano

\section{Sâm Ghavami}

\section{OpenEdition \\ Journals}

Édition électronique

URL : https://journals.openedition.org/jsa/19370

DOI : 10.4000/jsa. 19370

ISSN : $1957-7842$

\section{Éditeur}

Société des américanistes

Édition imprimée

Date de publication : 30 septembre 2021

Pagination : 45-76

ISSN : 0037-9174

Référence électronique

Sâm Ghavami, « Entre Mochica et Lambayeque : retracer les identités de la période Transitionnelle dans les empreintes du quotidien », Journal de la Société des américanistes [En ligne], 107-1 | 2021, mis en ligne le 30 septembre 2021, consulté le 03 septembre 2022. URL : http://journals.openedition.org/ jsa/19370 ; DOI : https://doi.org/10.4000/jsa.19370 


\title{
Entre Mochica et Lambayeque : retracer les identités de la période Transitionnelle dans les empreintes du quotidien
}

\author{
Sâm GHAVAMI *
}

\begin{abstract}
À la suite de l'effondrement de la structure sociopolitique mochica au IX ${ }^{\mathrm{e}}$ siècle de notre ère, l'affluence de nouvelles identités stylistiques d'influence huari a redéfini le paysage socioculturel de la côte nord du Pérou. Cet article propose d'évaluer la nature des relations qu'auraient entretenues les sites archéologiques de San José de Moro et de Huaca Bandera pendant la période Transitionnelle, à travers une analyse comparative de la céramique domestique. L'analyse céramographique comprend une étude minéralogique de la pâte au moyen d'un microscope numérique puis une caractérisation des éléments chimiques à l'aide d'un microscope électronique à balayage (MEB) dans le but de reconnaître des caractéristiques de production communes entre les deux sites. [Mots-clés : archéologie andine, Mochica, période Transitionnelle, archéométrie, technologie céramique, potiers itinérants.]
\end{abstract}

Between Mochica and Lambayeque: retracing the identities of the Transitional period through everyday life imprints. Following the collapse of the Mochica sociopolitical structure in the 9th century $\mathrm{AD}$, the affluence of new stylistic identities of Huari influence redefined the socio-cultural landscape of the north coast of Peru. This article proposes to assess the nature of the relationships that the archaeological sites of San José de Moro and Huaca Bandera would have maintained during the Transitional Period, through a comparative analysis of domestic ceramics. Ceramographic analysis includes a mineralogical study of the paste using a digital microscope and a characterization of the chemical elements using a Scanning Electron Microscope (SEM) in order to recognize production characteristics common to both sites. [Key words: Andean archeology, Mochica, Transitional Period, archaeometry, ceramic technology, swallow potters.]

Entre Mochica y Lambayeque: rastreando las identidades del periodo Transicional en las huellas del cotidiano. Tras el colapso de la estructura sociopolítica Mochica durante el siglo IX d.C., la afluencia de nuevas identidades estilísticas de influencia Huari redefinió el paisaje sociocultural de la costa norte del Perú. Este artículo propone evaluar la naturaleza de las relaciones que los sitios arqueológicos de San José

* Boursier FNS - Doc.CH, Sciences des religions, Faculté des Lettres, Université de Fribourg, Suisse [sam.ghavami@unifr.ch]. 
de Moro y Huaca Bandera habrían mantenido durante el período Transicional, a través de un análisis comparativo de la cerámica doméstica. El análisis ceramográfico incluye un estudio mineralógico de la pasta usando un microscopio digital y una caracterización de los elementos químicos usando un microscopio electrónico de barrido (MEB) con el fin de reconocer las características de producción común a ambos sitios. [Palabras clave: arqueología andina, Mochica, período Transicional, arqueometría, tecnología cerámica, alfareros golondrinos.]

Les recherches archéologiques dirigées sur la côte nord du Pérou font état d'une période de transition qui aurait suivi l'effondrement de la culture mochica durant le $\mathrm{IX}^{\mathrm{e}}$ siècle de notre ère et occasionné l'origine de la culture lambayeque ${ }^{1}$. Le site de San José de Moro dans la vallée de Jequetepeque est emblématique de ce bouleversement, car on y observe la lente disparition de l'expression artistique mochica simultanément à l'apparition d'une multitude de styles céramiques qui témoignent de la convergence d'autant de nouvelles identités (Rucabado-Yong 2006, 2008; Rucabado-Yong et Castillo 2003). Bien que l'on se soit principalement basé sur les études de cette région pour définir les aspects constitutifs de la phase transitionnelle, on constate depuis peu la prolifération de découvertes archéologiques semblables provenant des vallées de Lambayeque, de La Leche et de Motupe (Bracamonte Lévano 2015; Curo Chambergo 2013). Parmi les plus notables, le site monumental de Huaca Bandera, considéré comme l'un des derniers bastions mochica, présente des types céramiques d'une similarité étonnante avec ceux de San José de Moro (Curo Chambergo 2013). Ces récentes investigations ouvrent la voie à une compréhension plus globale de ce phénomène complexe, encore peu étudié audelà de la vallée de la Jequetepeque, puisqu'elles nous permettent de comparer ses différentes manifestations.

Cet article propose d'étudier avec plus d'attention les importantes similitudes stylistiques rencontrées sur des artefacts des sites archéologiques de cette période que les chercheurs assimilent à une « crise identitaire ». Ainsi, nous tenterons de comprendre les mécanismes qui ont permis l'expression de comportements analogues afin de clarifier la dynamique des relations sociopolitiques dans un contexte politique fragmenté. En outre, l'objectif est d'évaluer la nature des relations et le degré d'interaction entre le site archéologique de

1. Cet article est une synthèse du mémoire de Master de l'auteur en archéologie andine intitulé Rastreando identidades Post Mochica, soutenu en 2015 à la Pontificia Universidad Católica del Perú (PUCP) sous la direction du professeur Julio Rucabado-Yong. Plusieurs modifications et ajouts y ont été amenés. Nous utilisons la majuscule lorsque nous nous référerons à la période Transitionnelle pour rester fidèle à la nomenclature utilisée par les chercheurs en langue espagnole. L'adjectif a été substantivé puisque le «Transitionnel» se réfère uniquement à la période de transition issue de l'effondrement mochica sur la côte nord du Pérou. 
San José de Moro, situé dans la vallée de Jequetepeque-Chamán, et celui de Huaca Bandera, localisé dans la vallée de La Leche-Motupe, à la fin de la période Mochica tardive et le début de la phase « Transitionnelle », grâce à une analyse comparative de la production céramique. Celle-ci inclut une première observation des caractéristiques externes depuis une classification des types morphologiques présents sur les deux sites. Nous avons ensuite procédé à une analyse exhaustive d'un échantillon de dix fragments de chaque site sur lequel deux techniques archéométriques ont été appliquées : une étude optique de la texture de la pâte au microscope numérique et une caractérisation des éléments chimiques de la pâte à l'aide d'un microscope électronique à balayage (MEB). Le but du premier examen optique consiste à reconnaître une tradition technologique, c'est-à-dire une « recette de production » propre à un site ou à un type céramique. En second lieu, la caractérisation des pâtes nous permet de définir des comportements analogues dans la gestion des ressources (argiles et dégraissants) dans l'idée de démontrer si les similitudes morphologiques sont dues, ou non, à l'usage de matières premières identiques. Le cas échéant, nous pourrions soutenir l'hypothèse d'un réseau de distribution de la céramique ou même d'une interaction directe entre les deux sites.

La contribution de ce travail peut s'évaluer sur deux aspects. Le premier est archéologique, puisqu'il propose d'élargir le débat sur la phase transitionnelle aux « nouveaux acteurs » de la région, dont les récentes découvertes ont manifesté le rôle clé. Le deuxième aspect est méthodologique, puisqu'il s'inscrit dans une approche pluridisciplinaire qui combine l'analyse du mobilier archéologique domestique avec des techniques archéométriques ainsi que la discussion des résultats à la lumière des récents travaux ethnographiques (Druc 1996, 2013 ; Ramón Joffré 2008, 2011, 2013). Ce dernier point est fondamental étant donné que le mobilier utilitaire a trop souvent été écarté du débat archéologique sur les expressions matérielles de l'identité au profit du mobilier prestigieux, plus attrayant et plus facilement interprétable. Les changements observés dans les objets du quotidien sont discrets mais révèlent néanmoins de précieux indices concernant l'évolution des comportements et des pratiques sociales, sousjacentes pendant les périodes de transformation. L'équation « style décoratif ou technique = identité du producteur-consommateur » est, en outre, réévaluée par la grande diversité de contextes de production spécifiques aux artisans andins.

\section{La période Transitionnelle}

Les travaux pionniers de l'archéologie nord-péruvienne ont tissé un lien héréditaire, presque génétique, entre les Mochica et leurs descendants Lambayeque et Chimú (Larco 1948; Lavalle 1989; Rowe 1948). Or, si l'on supposait l'existence d'un passage d'une culture à l'autre, le contexte de ces transformations restait confus. Les premiers indices proviennent des sources ethnohistoriques 
selon lesquelles le bouleversement se serait subitement produit avec l'arrivée épiphanique sur les côtes du Pacifique d'un héros fondateur accompagné de sa cour (Cabello de Balboa 1951 [1586]). C'est en désirant attester archéologiquement de la véracité du récit mythique de Naimlap que Christopher Donnan entreprit la fouille du site Chotuna-Chornancap au début des années 1980, sans résultats probants (Donnan 1990). Uniques témoins des évènements du passé, les vestiges archéologiques qui portent encore les stigmates de leur temps racontent pour leur part une autre histoire: celle d'un changement progressif et incertain.

Que s'est-il passé dans le désert semi-aride de la côte septentrionale du Pérou entre le milieu du $\mathrm{IX}^{\mathrm{e}}$ siècle et début du $\mathrm{X}^{\mathrm{e}}$ siècle de notre ère? Du point de vue de l'archéologie, la transition s'observe tout d'abord avec l'apparition de plusieurs styles épigones mélangeant des symboles et des traditions technologiques multiples qui annoncent les canons à venir. C'est le cas des styles céramiques proto-Chimú et tricolore exhumés parmi les offrandes de contextes funéraires dans la région de Trujillo, ancien centre civico-cérémoniel majeur de la culture mochica, ou encore de la céramique de style early Sicán récupérée sur le site de Batán Grande, dans la région de Lambayeque (Donnan et Mackey 1978; Shimada 1981, 1985). Dans les vallées de Lambayeque et de La Leche, la phase correspondante est éponyme du style et se caractérise, selon Izumi Shimada (1981, p. 39), par un aspect transitionnel puisqu'elle résulte de l'effondrement de l'État Moche V à Pampa Grande et montre les premiers signes de l'ascension d'une nouvelle élite dans la forêt de Pomac. Pour l'archéologue, cet épisode historique correspond à la mise en place de formes sociales plus institutionnalisées et de profondes modifications des modes de pensée.

Toutefois, ce sont les découvertes faites à San José de Moro durant ces deux dernières décennies qui ont permis de scruter l'évolution des pratiques sociales et des représentations collectives dans cette époque de grands changements (Castillo 2001, 2012; Rucabado-Yong 2006, 2008; RucabadoYong et Castillo 2003). Situé sur la rive droite de la vallée de Jequetepeque, San José de Moro est un cimetière d'élite et un centre cérémoniel de la culture mochica, occupé continuellement pendant presque 1000 ans, entre le $\mathrm{V}^{\mathrm{e}}$ et $\mathrm{XV}^{\mathrm{e}}$ siècle apr. J.-C. (Figure 1). Les nombreuses sépultures exhumées dans la plaine de ce site ont permis de discerner différentes pratiques mortuaires, qui ont aidé à reconstruire les traditions funéraires des Mochica et des occupations successives Lambayeque et Chimú dans la région. La particularité de San José de Moro réside dans la découverte de véritables mausolées souterrains dédiés pour la plupart à des dignitaires féminins ayant régné à la période Mochica tardive. Ces somptueuses chambres funéraires étaient aménagées de manière à contenir la dépouille d'un ou plusieurs individus accompagnés par de multiples offrandes, parmi lesquelles les fameuses « lignes fines ${ }^{2}$ » et des modèles

2. Les « lignes fines du Mochica tardif » (LFMT) sont des bouteilles à anse-étrier en céramique où l'on a représenté avec extrêmement de finesse et un style presque baroque 
Entre Mochica et Lambayeque : retracer les identités de la période Transitionnelle

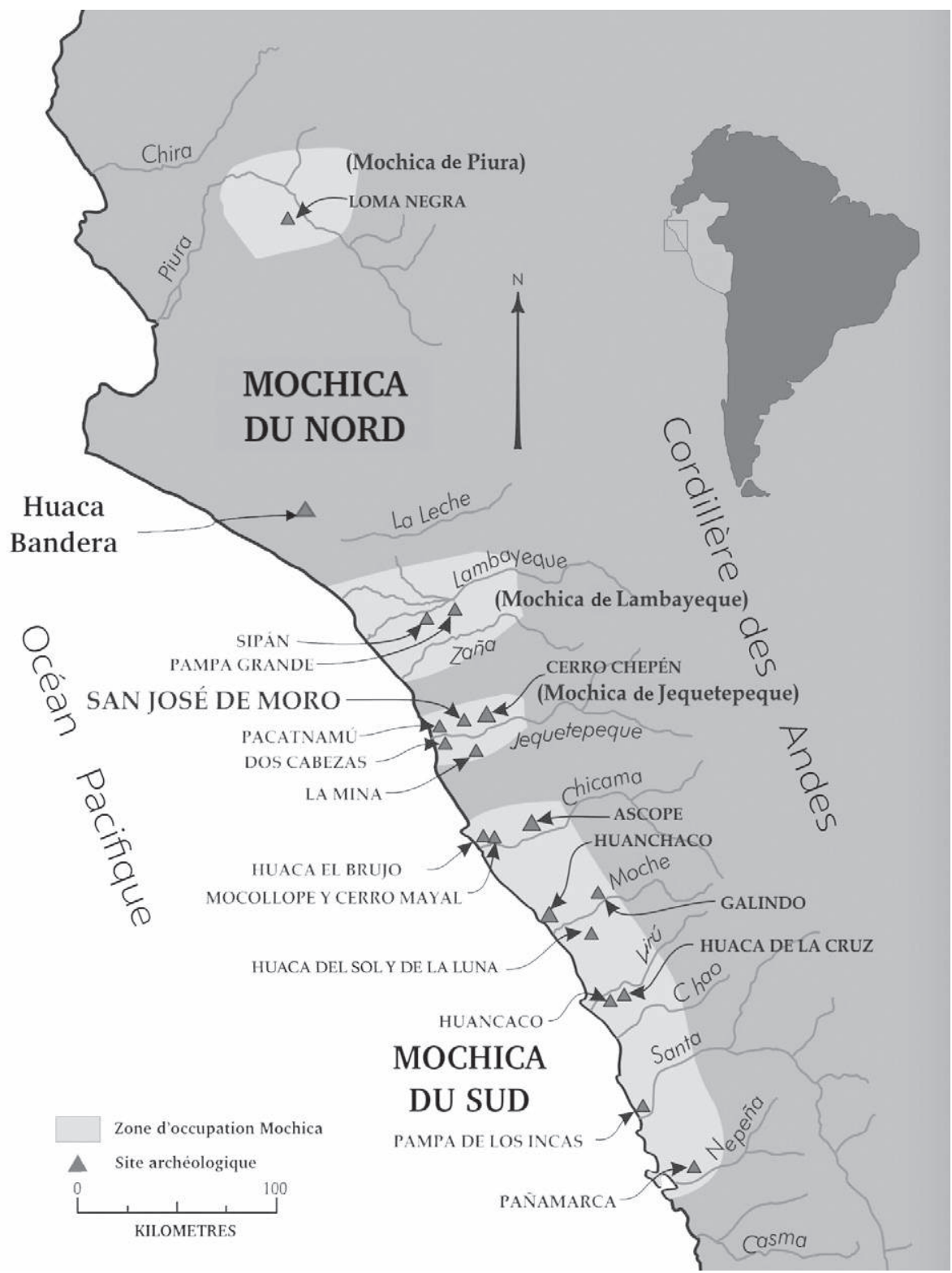

Fig. 1 - Carte du territoire mochica et localisation des sites mentionnés (archives PASJM)

de complexes scènes mythiques illustrant les croyances mochica. La quasi-totalité des céramiques de «lignes fines » de cette période retrouvées in situ provient des tombes de San José de Moro. 
réduits de temples en terre crue (Castillo 2001). Regroupant comme nul autre endroit la mémoire sépulcrale de hauts dignitaires mochica, San José de Moro jouissait d'un prestige unique dans la vallée de Jequetepeque, et les nombreux indices de festivités rituelles témoignent de l'afflux important de convives participant régulièrement aux célébrations sur le site. C'est pourquoi, malgré le contexte social fragmenté par les multiples factions autonomes de la vallée de Jequetepeque durant le Mochica tardif, Castillo considère que San José de Moro aurait joué un rôle d'intégrateur politique régional occasionnel au gré des besoins des différents acteurs. Il qualifie cette incorporation épisodique de l'organisation politique « d'État opportuniste» (Castillo 2012).

À San José de Moro, la période Transitionnelle a été identifiée à partir d'analyses stylistiques, stratigraphiques et funéraires. Elle se situe entre l'effondrement de la structure sociopolitique mochica vers 850 apr. J.-C. et la conquête de la vallée de Jequetepeque par l'État lambayeque vers l'an 1000 apr. J.-C. La période se caractérise principalement par le manque de standardisation dans la production des céramiques de style mochica et, par conséquent, par une grande hétérogénéité et une liberté de styles céramiques d'influences huari et cajamarca retrouvées dans les contextes funéraires et les strates d'occupation (Rucabado-Yong 2008). En outre, deux styles proprement locaux, le postMochica et le proto-Lambayeque, subsistent ou se développent pendant cette phase. Le premier se rapporte à des céramiques de qualité intermédiaire reflétant la tradition mochica persistante, alors que le deuxième compte principalement des vases ornés par une synthèse de symboles religieux mochica et de signes de pouvoirs huari (Figure 2). Ce syncrétisme culturel a donné lieu à l'iconographie et aux symboles de l'expression matérielle de la culture lambayeque (Castillo et Rucabado-Yong 2003; Lavalle 1989). Selon Castillo, la disparition progressive de la culture matérielle d'élite et des sépultures typiques, ainsi que l'abandon de la production des « lignes fines » à anses-étriers du style mochica polychrome, emblématiques dans leur rôle de support de l'idéologie religieuse, présagent la chute définitive du pouvoir des Mochica dans cette région. L'auteur précise toutefois que l'élite n'aurait pas simplement disparu, mais que les groupes dominants se seraient restructurés (Castillo 2001, 2012; Rucabado-Yong et Castillo 2003). Cette lente réorganisation du paysage socioculturel est au cœur de la dynamique de la phase transitionnelle qui reflète non pas la substitution d'une identité à une autre, mais plutôt l'interaction de multiples identités dont les expressions culturelles se côtoient désormais dans des contextes archéologiques jadis exclusivement mochica.

La superposition de deux types de tombeaux a été déterminée stratigraphiquement lors des fouilles, ce qui a conduit les archéologues à diviser la période de transition en deux sous-phases (Castillo 2012). La sous-phase A se caractérise par des chambres funéraires rectangulaires très similaires à celles des prêtresses du Mochica tardif, et la sous-phase B présente quant à elle des 
Entre Mochica et Lambayeque : retracer les identités de la période Transitionnelle

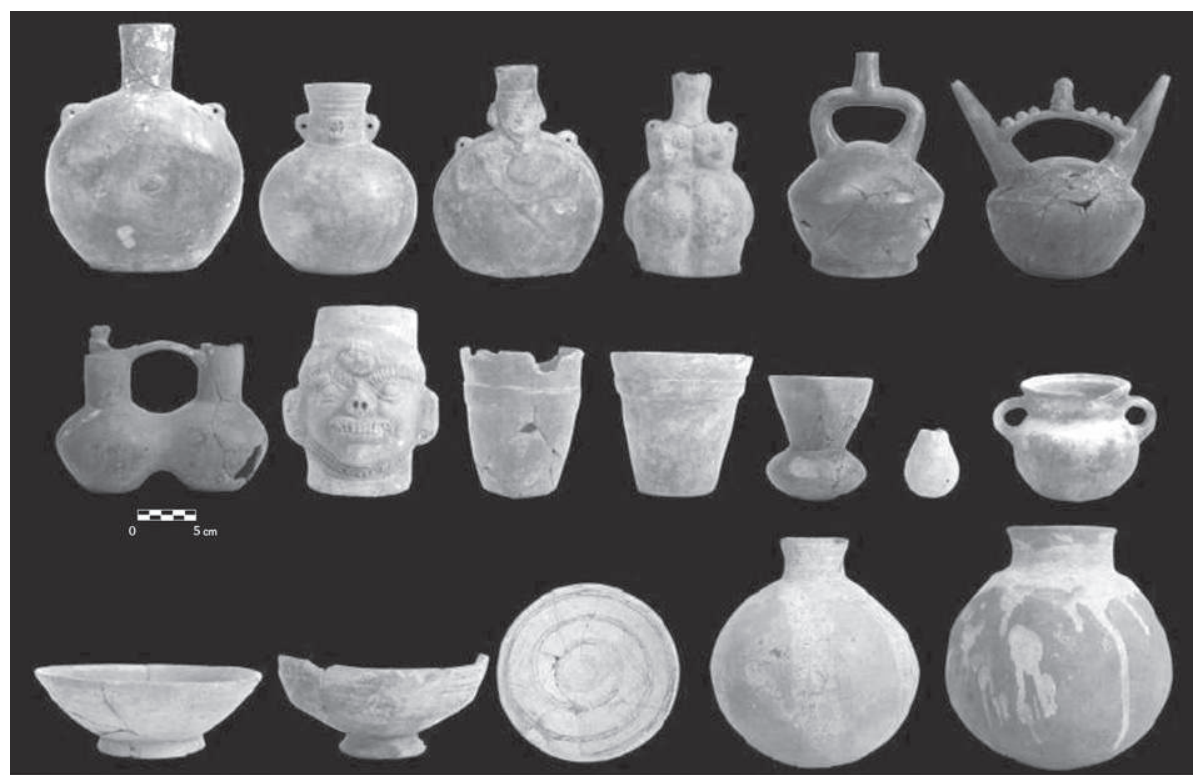

Fig. 2 - Ensemble de céramiques de styles post-Mochica, proto-Lambayeque et Cajamarca provenant des offrandes funéraires de la tombe M-U615 de la phase Transitionnelle A à San José de Moro (archives PASJM, Rucabado-Yong 2006)

coutumes funéraires modifiées puisque les chambres funéraires sont à la fois plus petites et contiennent un nombre d'individus et de styles céramiques plus élevés que celles de la période précédente. Les transformations relatives au Transitionnel B signalent une utilisation multigénérationnelle sous la forme de mausolées familiaux. Ceux-ci évoquent les chullpas des traditions andines adaptées au culte des ancêtres. Rucabado-Yong (2006) affirme que la réouverture et l'utilisation constante des tombes à l'occasion des funérailles auraient fait partie de la nouvelle stratégie de l'élite pour manifester une filiation directe avec ses ancêtres et légitimer ainsi son pouvoir. Cette pratique aurait soutenu la mise en place de nouveaux mécanismes idéologiques pour la transmission du pouvoir religieux pendant la phase de transition où tout porte à croire que de multiples traditions et idées auraient interagi. En effet, cette deuxième phase voit le pourcentage de céramique de style cajamarca augmenter considérablement dans les tombes, indiquant une possible participation directe des communautés andines aux cérémonies funéraires de San José de Moro (Rosas 2007).

Le complexe archéologique de Huaca Bandera est situé dans la partie supérieure de la vallée de La Leche, dans l'actuel district de Pacora (voir Figure 1). Le site est dominé par trois huacas majeures organisées par un réseau de canaux et d'enceintes fortifiées (Curo Chambergo 2013). Les caractéristiques stylistiques et d'occupation plaident en faveur d'un emplacement chronologique de Huaca 
Bandera pendant les périodes Mochica tardif C et Transitionnel A selon la nomenclature utilisée dans la vallée de Jequetepeque, ou la phase early Sicán selon celle employée dans les vallées de Lambayeque et de La Leche, c'està-dire à une approximation en chronologie absolue entre 800 et 900 apr. J.-C. La Huaca Bandera, éponyme du site, est située dans le secteur nord du complexe, au sein de l'enceinte fortifiée 1. Il s'agit de la plus grande pyramide tronquée du complexe, dotée d'une rampe centrale et présentant un motif architectural alambiqué. On parvient à l'accès principal par un passage emmuré de 60 mètres de long qui relie directement l'extérieur du complexe à la rampe centrale. La structure est composée de plusieurs plateformes similaires à des banquettes des deux côtés, qui pourraient s'apparenter à des trônes ou des autels selon Curo Chambergo (ibid.). De plus, le site est composé d'un vaste système de zones administratives avec des structures en forme de $U$, de zones domestiques et de grandes places souvent reliées à des plateformes pyramidales.

L'un des aspects singuliers de Huaca Bandera provient de la quantité de décorations murales qui révèle en partie l'identité des occupants. Les personnages et les scènes représentés sur les murs du complexe archéologique font allusion à une tradition profondément enracinée dans la culture mochica, comme si l'intention des occupants était de revendiquer leur héritage (Figure 3). En effet, on distingue la scène de « la présentation de la coupe » ainsi que l'omniprésence du panthéon mythique dans les nombreuses peintures murales et graffitis qui ornent le site (Curo Chambergo 2013). Il convient aussi de noter l'absence quasi totale de céramique de style cajamarca sur le site - un cas particulier pour la période - qui indiquerait peut-être une stratégie délibérée des dirigeants pour ne pas s'affilier à leurs voisins andins. Cette supposition est appuyée par l'emplacement géographique du site qui se situe loin de la cordillère des Andes, à l'orée de la plaine désertique de Sechura. À ce titre, il est intéressant de mettre en perspective les caractéristiques de Huaca Bandera que nous venons de mentionner avec les données obtenues lors des récentes fouilles archéologiques conduites sur le site de Santa Rosa de Pucalá dans la vallée de Lambayeque. Celles-ci ont mis à jour, pour la première fois sur la côte nord, une structure cérémonielle en « $\mathrm{D}$ », emblématique de la culture huari (Bracamonte Lévano 2019a). Cette découverte renforce l'idée d'une présence, voire d'un contrôle direct de groupes andins sur le territoire côtier pendant la période en question. D'autant plus que le site abonde justement en céramique de style cajamarca liée à cette même occupation suggérant une forte affinité avec les traditions andines, à l'inverse de Huaca Bandera. Cette discussion montre combien, face à la crise, la stratégie des dignitaires mochica pouvait radicalement varier selon les régions, sans doute en raison d'un paysage sociopolitique déjà fragmenté et décentralisé lors de la période Mochica tardive (Castillo 2001). C'est pourquoi nous devrions concevoir la transition comme un phénomène multidimensionnel et complexe qui aurait pris forme 


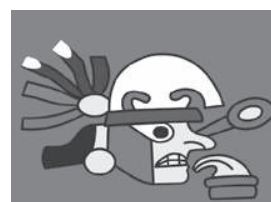

A

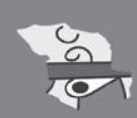

B

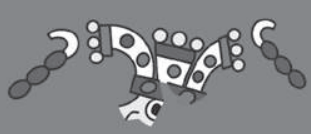

C

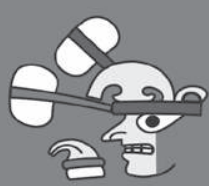

D

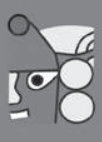

E

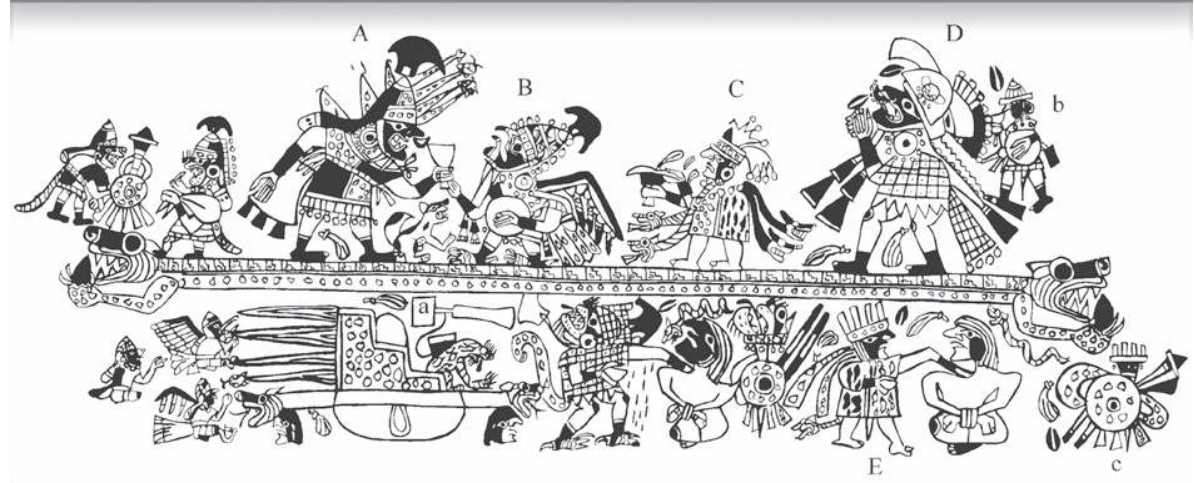

Fig. 3 - En haut, dessin de la peinture murale de Huaca Bandera faisant référence à la scène de la présentation de la coupe (archives PIAHB, Curo Chambergo 2013).

En bas, la scène en question dans l'iconographie mochica (Donnan et McClelland 1999).

au gré des réponses de chaque acteur face aux situations qui se présentaient à eux. Il n'y aurait donc pas « un passage » de Mochica à Lambayeque (voir McClelland 1990), mais plusieurs.

\section{Sur la relation entre le style et l'ethnicité}

En vue d'étudier la nature de la relation existant entre deux communautés qui partagent le même style, il nous paraît approprié de réexaminer les liens de corrélations entre les notions de style et d'ethnicité dans la littérature des sciences sociales. Il est en outre question « d'ethnicité » ou « d'identité ethnique » car c'est en s'évertuant à reconnaître les groupes humains dans le passé que les archéologues ont communément analysé les vestiges de la culture matérielle. Autrement dit, la répartition des styles culturels était traditionnellement considérée comme le reflet de l'espace de diffusion d'un groupe ethnique. D'autre part, l'ethnicité peut se concevoir comme un aspect constitutif d'une dimension plus générale qu'est « l'identité ». Ainsi, nous rencontrons une distinction entre différentes formes d'identité comme pourrait l'être l'identité politique ou l'identité religieuse, chacune soulignant une appartenance socioculturelle propre. 
Au tournant du $\mathrm{Xx}^{\mathrm{e}}$ siècle, les prémices de l'archéologie moderne ont développé une méthodologie scientifique qui a permis de légitimer un discours primordialiste en dépeignant l'ethnicité comme une notion héréditaire, stable et indépendante des relations avec d'autres groupes (Childe 1929; Geertz 1973; Kossinna 1911). Cependant, la supposée homogénéité des groupes humains selon le paradigme langue-race-culture était contestée par de nouvelles théories soulignant le caractère social de la construction de l'ethnicité. À l'inverse, les courants processuels perçoivent l'ethnicité comme flexible et variable, résultant de processus sociaux et politiques (Barth 1969; Díaz-Andreu et Lucy 2005 ; Jones 1996; Lucy 2005). Fredrik Barth soutient que c'est par le biais d'un phénomène d'interaction sociale - les processus sociaux d'inclusion et d'exclusion - que les groupes ethniques se définissent, créant de la sorte des frontières entre « nous » et « eux » (le we feeling). Selon lui, le fondement de la diversité ethnique est justement produit par l'interaction avec d'autres sociétés, et non par l'isolement géographique et culturel. L'identité ethnique n'est plus vue comme un reflet passif des normes culturelles, mais un aspect à part entière de l'organisation sociale qui inclut un entretien actif des frontières ethniques lors des interactions (Barth 1969). Il est intéressant de soulever ici que la temporalité des changements de paradigmes épistémologiques correspond à des changements plus globaux dans les régimes de régulation du social. En effet, l'idée que la culture matérielle soit directement liée au degré de " pureté » de l'ethnie en tant que groupe humain au profil racial prédéterminé est un a priori qui reflète un contexte propre au XIX ${ }^{\mathrm{e}}$ siècle lorsque la question de la consolidation de l'État-nation était prédominante et le nationalisme fort. De même que le changement de paradigme à une conception plurielle et fluide de l'ethnicité reflète le passage de l'État-nation à l'économie de marché comme principe régulateur de la société (Gauthier 2019).

La perspective émique de Barth met pourtant l'emphase sur le rôle communicatif de la culture matérielle. En effet, lors des interactions sociales, les frontières ethniques sont créées et maintenues moyennant le maniement de symboles explicites qui communiquent visuellement l'appartenance culturelle des acteurs. Il ne s'agit pas de la totalité de la culture matérielle mais seulement de certains éléments culturels que les acteurs considèrent comme pertinents et qui peuvent varier selon les groupes (Hodder 1982; Jones 1996, p. 28). Si les caractéristiques essentielles qui définissent l'ethnicité sont l'auto-attribution en plus de l'attribution par d'autres groupes, alors il est question d'intentionnalité et de conscience de soi. C'est effectivement ce que remarque Ian Hodder dans ses travaux ethnoarchéologiques en Afrique de 1'Est, qui avaient notamment pour but de vérifier les suppositions de la théorie de l'interaction sociale de Barth. L'auteur montre que la mesure dans laquelle la similitude culturelle se rapporte à l'interaction dépend des stratégies et des intentions des groupes en interaction et de la façon dont ils utilisent, manipulent et négocient les symboles 
matériels dans le cadre de ces stratégies (Hodder 1982, p. 185). C'est pourquoi nous pouvons inférer que les symboles manifestés pour revendiquer une identité particulière se retrouvent plus généralement sur des objets de qualité fine qui arborent des signes visuels caractéristiques. De plus, il peut être argumenté que la sélection de signes distinctifs pour délimiter des frontières entre groupes n'a pas uniquement vocation à revendiquer une appartenance ethnique. Celle-ci pourrait dans de nombreux cas mieux illustrer une stratégie d'ordre politique, de la même manière que le choix d'un certain style vestimentaire reflèterait la construction d'un statut social (Curta 2014, p. 2509). Le matériel culturel que nous analysons dans ce travail ne présente pour sa part pas de signes visuels forts puisqu'il appartient au mobilier utilitaire d'usage domestique. Ce groupe se distingue non pas par des « symboles ethniques » mais plutôt par ce que l'on pourrait nommer en contraste des « empreintes ethniques » puisqu'elles sont les traces laissées de manière involontaire lors du processus de production propre à la communauté qui fabrique l'objet. De ce fait, il convient d'examiner avec plus d'attention les modes de production afin de déceler les techniques analogues qui permettraient d'identifier des affinités particulières entre les différents groupes. En d'autres termes, l'analyse passerait d'une observation macroscopique au niveau microscopique. Par conséquent, nous sommes amenés à questionner le rôle que les artisans (potiers, métallurgistes ou tisserands) ont exercé dans la construction de l'identité ethnique du groupe par les pratiques au quotidien.

Cette question est à l'origine de récents travaux archéométriques et ethnoarchéologiques qui s'accordent à souligner l'importance des analyses du « style technique » comme réel indicateur de l'ethnicité (Druc 1996, 2013 ; Gosselain 2000; Jones 1996; Lucy 2005; Makowski et al. 2008 ; Ramón Joffré 2008, 2013). Comme le mentionne Gabriela Oré Menendez (2012, p. 13), "s'agissant de la production, le "comment" est devenu plus important que le "quoi" ». Cette approche indique que la tradition technologique d'une société fait partie intégrante de son identité - puisqu'il faut assister physiquement à sa fabrication pour l'apprendre - et que, par là même, elle en est l'aspect le plus stable. Le « style décoratif » est, quant à lui, variable et plus facile à imiter puisqu'il est un aspect superficiel et visible de tous. La tradition technologique concerne à la fois le type de matière première, son origine, la taille et l'abondance des minéraux contenus dans les argiles et les recettes reflétant les processus de fabrication, comme le façonnage, le traitement des surfaces, le décor ou la cuisson. Les caractéristiques des pâtes céramiques peuvent indiquer des productions locales ou étrangères, alors que leur distribution reflèterait les réseaux de commerce et les frontières sociales entre différents groupes (Druc 2013, p. 486). Les travaux ethnographiques d'Isabelle Druc (1996, 2013) ont montré justement que les potiers pouvaient modifier à leur guise les formes et les décorations, mais étaient beaucoup moins susceptibles de changer leur technique de production. 
L'approche ethnoarchéologique se concentre dès lors sur les étapes de production des objets culturels, communément appelées la " chaîne opératoire ", dont le processus contribuerait à déterminer l'identité ethnique. En effet, de la sélection de la matière première à sa production et sa distribution jusqu'à son utilisation, se succède une série d'actions qui laisse une signature caractéristique sur le résultat final. Identifier cette signature revient à déterminer l'identité du groupe du point de vue de son savoir-faire et de ses pratiques. Ces « techniques du corps » réunissent l'ensemble des actions répétées comme des manifestations de logiques culturelles qui sont transmises dans la société sous la forme de traditions (Mauss 1934). L'identité ethnique pourrait se concevoir dès lors comme le résultat de l'intersection des dispositions de l'habitus et des conditions sociales qui existent dans un contexte historique particulier (Bourdieu 1972; Curta 2014). Aussi, les techniques scientifiques utilisées de nos jours sont multiples pour détecter les empreintes laissées par ces actions. C'est le cas de la caractérisation minéralogique et élémentaire des pâtes pour la production des céramiques. Ces analyses physico-chimiques permettent de nous informer sur l'origine des matières premières, de définir les différentes productions d'un même site ou de les comparer avec d'autres, afin de mettre en évidence des circulations de récipients dans une zone déterminée. C'est pourquoi les méthodes archéométriques sont plus à même d'analyser ces « empreintes ethniques » caractéristiques dans la production des objets du quotidien.

\section{Analyses des échantillons céramiques}

Dans le but de comparer la production des céramiques retrouvées sur les sites de San José de Moro et de Huaca Bandera, nous sommes partis d'une observation initiale et générale d'un échantillonnage représentatif des céramiques sur chaque site, guidé par une comparaison morphologique et décorative du matériel récupéré. Autrement dit, nous nous sommes appuyés sur une partie des caractéristiques externes et visibles du mobilier culturel. C'est à partir de cette observation macroscopique que nous avons ensuite approfondi l'analyse pour réaliser une étude microscopique des pâtes utilisées dans la fabrication des céramiques, comme un moyen de se plonger dans la caractérisation des aspects constitutifs moins visibles de la composition des pâtes et des argiles, mais qui définissent plus finement la production différenciée de poteries. En concertation avec Manuel Curo Chambergo, l'archéologue de Huaca Bandera, notre étude s'est limitée au mobilier utilitaire. En tenant compte de cette restriction, il nous a fallu réélaborer notre méthodologie et ne sélectionner que des fragments de céramique non décorée. Nous avons donc laissé de côté les céramiques qui présentaient un traitement soigné, comme le polissage, et des décorations en ronde-bosse ou picturales fines, les potentiels symboles culturels. Cette limitation n'a donné que plus de valeur à notre étude, puisque le matériel d'usage 
quotidien, de qualité plus grossière, a souvent été délaissé lors des analyses archéométriques, au profit du mobilier prestigieux. De plus, cela contribua à concentrer adéquatement notre étude sur les caractéristiques de production, plus à même de retracer les liens d'ordre ethnique. Ainsi, les résultats obtenus ont permis une caractérisation préliminaire des éléments constitutifs de la composition des pâtes du mobilier domestique dont la variation des recettes de fabrication a pu mettre en lumière des comportements analogues aidant à déceler de possibles réseaux de production.

En premier lieu, nous avons donc réalisé une typologie morpho-fonctionnelle du mobilier céramique de Huaca Bandera, qui a également tenu compte du traitement des surfaces et de l'atmosphère de cuisson des objets. Notre étude s'est concentrée sur le secteur nord 1, un tertre de logement situé à environ 60 mètres au nord de la huaca principale, où 908 fragments de céramique diagnostique ont été collectés. De ce nombre, nous avons soustrait le matériau lié au niveau superficiel ainsi que la céramique de qualité fine, comptabilisant un total de 489 tessons (voir Ghavami 2016 pour plus de détails concernant la typologie). Cette observation générale a permis de constater la présence des différents types de céramique sur le site - principalement des marmites et des cruches - dans l'idée de comparer cet ensemble avec les catégories morpho-fonctionnelles provenant des contextes de l'occupation transitionnelle de San José de Moro (voir Cusicanqui Marsano 2010; Muro-Ynoñan 2009). Le résultat de la classification est univoque: nous trouvons à San José de Moro une correspondance parfaite pour les 33 types des 10 catégories de céramiques récupérées dans le secteur nord 1 de Huaca Bandera (voir Figure 4). Ce constat nous incite à observer de plus près les caractéristiques de manufacture et la provenance des matières premières, afin de déceler une possible connexion entre les deux sites, comme un réseau de production. Pour ce faire, nous avons procédé à une caractérisation minérale et chimique de la céramique de Huaca Bandera et de San José de Moro à partir de l'analyse de 20 échantillons (Figure 5). Nous avons sélectionné 10 fragments de l'ensemble des céramiques utilitaires du secteur nord 1 de Huaca Bandera, dans l'idée que le groupe serait représentatif de l'hétérogénéité des catégories de formes et de styles techniques trouvés sur le site. Puis, dans le but de comparer adéquatement les types, nous avons choisi les fragments de San José de Moro les plus similaires liés à la fois à la forme, au traitement, à la cuisson et aux strates d'occupation: en d'autres termes, leurs équivalents. Si le nombre d'échantillons analysés est relativement faible, il permet toutefois une comparaison et une caractérisation préliminaire de la production de ce type de céramiques. Ce premier effort devra indéniablement inspirer de futurs travaux archéométriques permettant la comparaison des productions céramiques sur davantage de sites de la côte nord pour assurer une meilleure compréhension des relations interrégionales pendant la période Transitionnelle (conformément à ce qu'a déjà entrepris Bracamonte Lévano 2019b). 


\begin{tabular}{|c|c|c|c|}
\hline \multicolumn{2}{|c|}{ Huaca Bandera } & \multicolumn{2}{|c|}{ San José de Moro } \\
\hline Référence & Description & Référence & Description \\
\hline $\begin{array}{l}\text { HB } 1 \text { LXXIII P"” } \\
(78)\end{array}$ & $\begin{array}{l}\text { Fragment (frg.) } \\
\text { d'un plat de cuisson } \\
\text { réducteur et poli }\end{array}$ & SJM 1 Frg. M-U615 & $\begin{array}{l}\text { Frg. d'un plat de } \\
\text { cuisson réducteur } \\
\text { et poli }\end{array}$ \\
\hline $\begin{array}{l}\text { HB } 2 \text { LXXIII R"” } \\
(49)\end{array}$ & $\begin{array}{l}\text { Frg. d'un pot avec } \\
\text { une poignée en } \\
\text { forme de bras }\end{array}$ & $\begin{array}{c}\text { SJM } 2 \text { A20 - C7 } \\
-47\end{array}$ & $\begin{array}{l}\text { Frg. d'un pot avec } \\
\text { une poignée en } \\
\text { forme de bras }\end{array}$ \\
\hline $\begin{array}{c}\text { HB } 3 \text { LXXI Q"” } \\
(63)\end{array}$ & $\begin{array}{l}\text { Frg. de la poitrine } \\
\text { d'une figurine } \\
\text { féminine }\end{array}$ & $\begin{array}{l}\text { SJM } 3 \text { A } 45-\text { C2 } \\
\quad-\text { Fc13- } 72\end{array}$ & $\begin{array}{l}\text { Frg. de la poitrine } \\
\text { d'une figurine } \\
\text { féminine }\end{array}$ \\
\hline $\begin{array}{c}\text { HB } 4 \text { LXXI Q"” } \\
(51)\end{array}$ & $\begin{array}{l}\text { Frg. d'un pot globu- } \\
\text { laire à lèvre inversée }\end{array}$ & SJM 4 A $20-$ C6 - 2 & $\begin{array}{l}\text { Frg. d'un pot globu- } \\
\text { laire à lèvre inversée }\end{array}$ \\
\hline $\begin{array}{c}\text { HB } 5 \text { LXXIII R"” } \\
(47)\end{array}$ & $\begin{array}{l}\text { Frg. d'une bouteille } \\
\text { flacon }\end{array}$ & $\begin{array}{l}\text { SJM } 5 \text { A } 45-\mathrm{C} 2 \\
-\mathrm{Fc} 014-85\end{array}$ & $\begin{array}{l}\text { Frg. d'une bouteille } \\
\text { flacon réducteur }\end{array}$ \\
\hline $\begin{array}{c}\text { HB } 6 \text { LXXIII Q"” } \\
(82-83)\end{array}$ & $\begin{array}{l}\text { Frg. d'une cruche à } \\
\text { col droit }\end{array}$ & $\begin{array}{c}\text { SJM } 6 \text { A } 45-\text { C3 } \\
- \text { R13 - Fc02-29 }\end{array}$ & $\begin{array}{l}\text { Frg. d'une cruche à } \\
\text { col droit }\end{array}$ \\
\hline $\begin{array}{c}\text { HB } 7 \text { LXXIII Q"” } \\
(73)\end{array}$ & $\begin{array}{l}\text { Frg. d'un pot } \\
\text { avec décoration } \\
\text { paleteada }\end{array}$ & $\begin{array}{l}\text { SJM } 7 \text { A45 - C2 } \\
- \text { Fc12-140 }\end{array}$ & $\begin{array}{l}\text { Frg. d'un pot } \\
\text { avec décoration } \\
\text { paleteada }\end{array}$ \\
\hline $\begin{array}{l}\text { HB } 8 \text { LXXIII P", } \\
(46)\end{array}$ & $\begin{array}{l}\text { Frg. d'un pot à } \\
\text { bord droit et lèvre } \\
\text { convexe }\end{array}$ & $\begin{array}{l}\text { SJM } 8 \text { A } 45-C 3 \\
- \text { Fc05- } 14\end{array}$ & $\begin{array}{l}\text { Frg. d'un pot à } \\
\text { bord droit et lèvre } \\
\text { convexe }\end{array}$ \\
\hline $\begin{array}{c}\text { HB } 9 \text { LXXIII P"” } \\
(77-78)\end{array}$ & $\begin{array}{l}\text { Frg. d'une cruche à } \\
\text { col-visage }\end{array}$ & $\begin{array}{l}\text { SJM } 9 \text { A20 - C3 } \\
-610\end{array}$ & Frg. d'une cruche \\
\hline $\begin{array}{c}\text { HB } 10 \text { LXXI Q"” } \\
(64)\end{array}$ & Frg. d'une râpe & $\begin{array}{c}\text { SJM } 10 \text { A21-C3 } \\
-\mathrm{N} 1-43\end{array}$ & Frg. d'une râpe \\
\hline
\end{tabular}

Fig. 4 - Typologie comparative des céramiques domestiques de Huaca Bandera et de San José de Moro. Exemples de la correspondance parfaite des types de pots (Ghavami 2016)

L'objectif de cette étude est également de fournir des informations sur la production céramique des sites étudiés. La composition minéralogique d'un récipient reflète un mode de production et une zone d'acquisition. Les caractéristiques minérales et la composition élémentaire des céramiques doivent donc être identifiées pour permettre la comparaison, la classification et le groupement de céramiques de même production (Druc 1996). En suivant cette méthodologie, nous avons réalisé une analyse minéralogique de base à partir de photos prises avec un microscope numérique Koolertron 8-LED USB sur des angles nouvellement sectionnés de chaque fragment. Celle-ci consiste, d'abord, en une observation qualitative, afin d'identifier et de reconnaître la composition 
Entre Mochica et Lambayeque : retracer les identités de la période Transitionnelle

des inclusions, leur taille relative, leur forme, leur altération et leur disposition dans la pâte. Elle se complète, ensuite, par une analyse quantitative qui inclut le calcul du nombre d'inclusions en fonction du type minéral ou lithique. Ces différentes observations permettent d'identifier, dans la mesure du possible, les groupes de même composition et les céramiques de composition atypique (ibid., p. 58). Les résultats obtenus ont ensuite pu être comparés aux données typologiques et chimiques pour nous aider à observer une potentielle corrélation entre une tradition technologique et une catégorie typologique sur chaque site.

L'analyse de la composition minéralogique de base nous a permis de classer les échantillons en six groupes renvoyant à différentes caractéristiques de la texture des pâtes et de ses composants. Cependant, si l'échantillonnage est trop petit pour établir une réelle corrélation indiquant une tradition technologique, elle nous permet, néanmoins, de proposer une caractérisation préliminaire des pâtes. En premier lieu, il s'avère que la matrice argileuse varie moins que le dégraissant. En effet, les potiers ont ajouté dans l'argile une grande quantité de dégraissants composée d'une importante variété granulométrique et d'autres

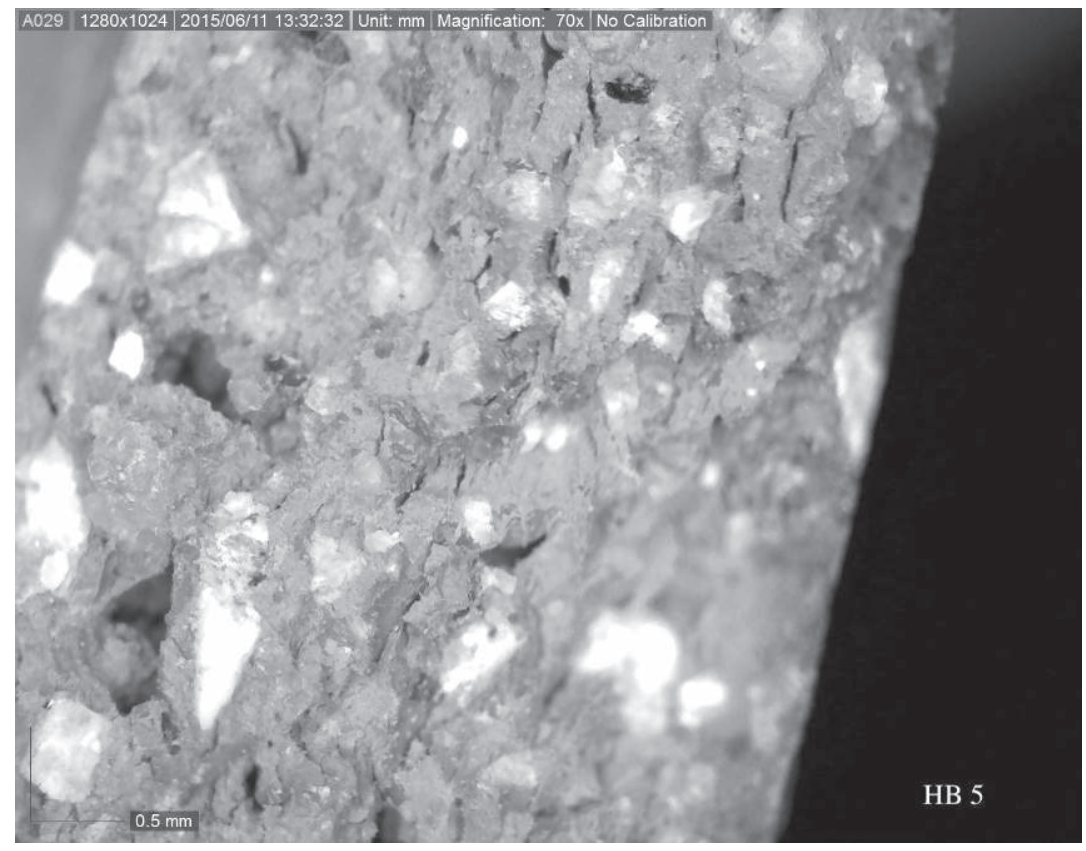

Fig. 6 - Images en coupe au microscope numérique des échantillons du groupe 1 . La pâte des céramiques présente des pores principalement élargis de couleur beige à rouge-orangé. Les inclusions sont distribuées de manière relativement homogène comprenant des quartz, poly quartz, feldspaths et probablement des mafiques occasionnels (pour plus de détails, voir Ghavami 2016) 


\section{Sâm GHavami}

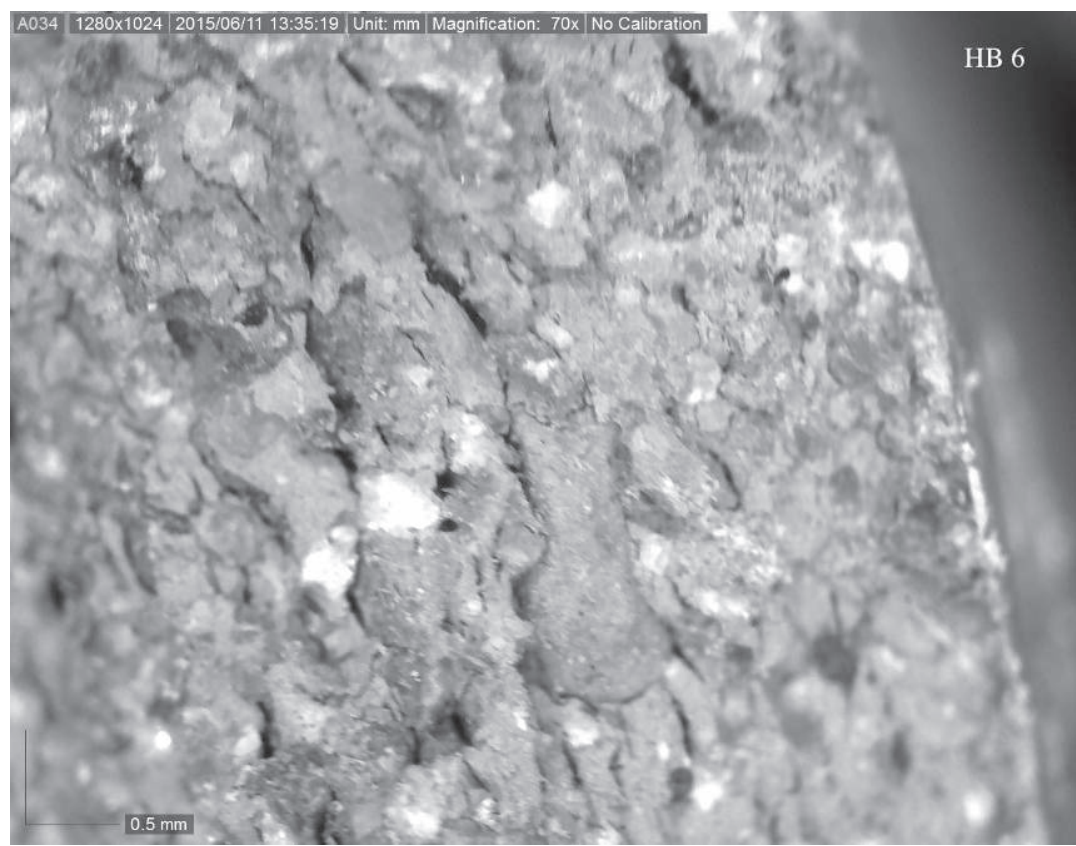

Fig. 7 et 8 - Images en coupe au microscope numérique des échantillons du groupe 1. La pâte des céramiques présente des pores principalement élargis de couleur beige à rouge-orangé. Les inclusions sont distribuées de manière relativement homogène comprenant des quartz, poly quartz, feldspaths et probablement des mafiques occasionnels (pour plus de détails, voir Ghavami 2016)

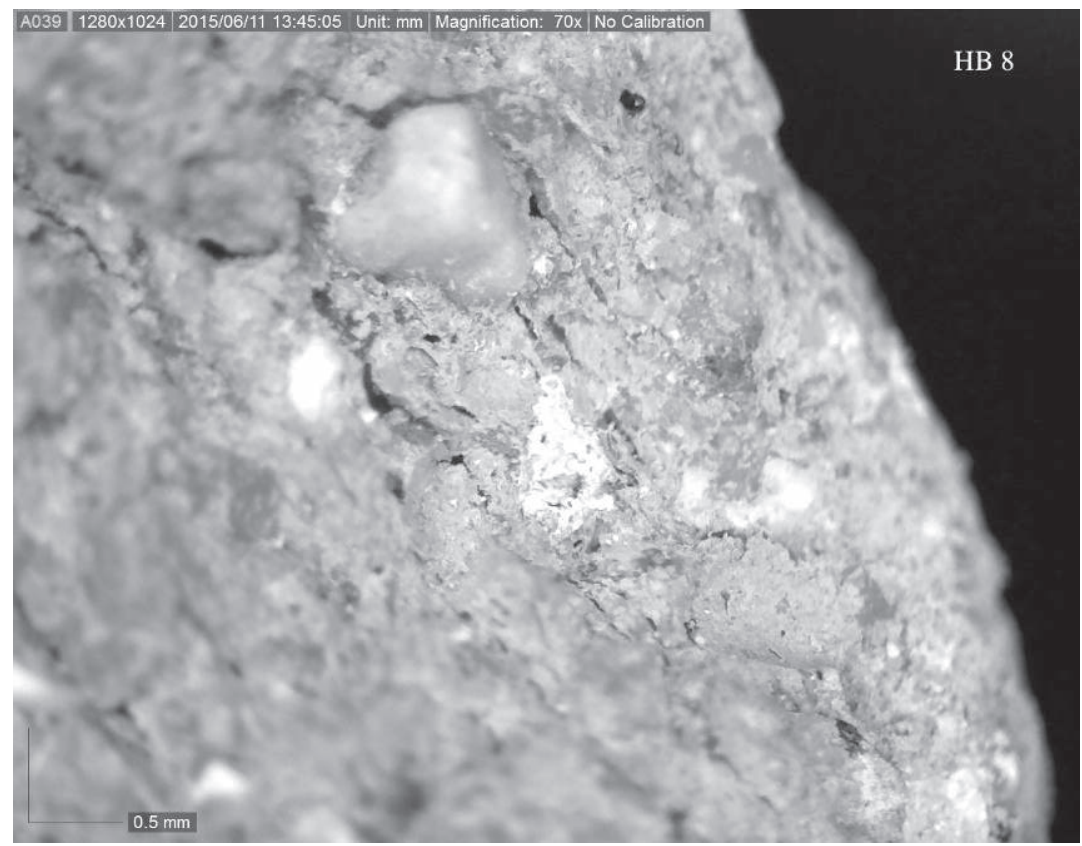


Entre Mochica et Lambayeque : retracer les identités de la période Transitionnelle

éléments distincts. On reconnaît principalement du quartz et du feldspath avec des proportions variables dans chaque fragment (Figures 6 à 8). Ces caractéristiques seraient typiques de la production de céramiques domestiques si l'on se fie aux résultats des études archéométriques dans la vallée de Jequetepeque. Rohfritsch (2006, p. 46) a, en effet, souligné que les céramiques de qualité fine étaient formées par un dégraissant constitué d'une granulométrie dense d'inclusions moyennes relativement homogènes, tandis que les céramiques domestiques étaient généralement constituées par un dégraissant composé d'inclusions de plus grandes proportions (2-3 $\mathrm{mm}$ ) et aussi d'une granulométrie beaucoup plus variable. Ce deuxième cas est précisément ce que l'on observe dans nos échantillons. Les minéraux les plus récurrents que nous avons pu reconnaître sont des lithoclastes de types mafiques et les grès. De manière générale, les fragments présentent un fort caractère côtier, probablement composés d'argile limoneuse (Druc, com. pers. 2015). Ces propositions doivent, néanmoins, être considérées comme une première approximation de la caractérisation des pâtes de ces céramiques, puisqu'une analyse pétrographique formelle et complète serait nécessaire pour définir les composants de manière plus certaine.

\section{L'analyse de la composition chimique des pâtes}

Les analyses de la composition des principaux éléments chimiques de la pâte ont été effectuées à l'aide d'un microscope électronique à balayage (MEB) - Quanta 650-FEI, avec détecteur EDAX Team (EDS) à fort grossissement jusqu'à une surface de $1 \mu \mathrm{m}^{2}$ en mode spot. En premier lieu, les résultats des analyses élémentaires proviennent de la moyenne de cinq zones de $40 \mu \mathrm{m}^{2}$ de chaque échantillon dans le but d'observer uniquement les variations de la composition chimique de la matrice argileuse, sans tenir compte des inclusions (Figures 9 et 10). Nous avons ensuite procédé à l'analyse de la zone complète de $600 \mu \mathrm{m}^{2}$ qui comprend la matrice argileuse ainsi que les inclusions naturelles et celles du dégraissant. Les résultats sont exprimés en pourcentages d'oxydes (Figure 11; pour plus de détails, voir Ghavami 2016).

Fig. 9 - Tableau de la composition élémentaire des matrices argileuses du groupe de Huaca Bandera (en \% d'oxydes; PM : moyenne; Ghavami 2016)

\begin{tabular}{|c|c|c|c|c|c|c|c|c|c|c|}
\hline Échantillon & $\mathbf{N a}_{2} \mathbf{O}$ & $\mathbf{M g O}$ & $\mathbf{A l}_{2} \mathbf{O}_{\mathbf{3}}$ & $\mathbf{S i O}_{2}$ & $\mathbf{P}_{2} \mathbf{O}_{\mathbf{5}}$ & $\mathbf{K}_{2} \mathbf{O}$ & $\mathbf{C a O}$ & $\mathbf{T i O}_{2}$ & $\mathbf{F e}_{2} \mathbf{O}_{3}$ & Total \\
\hline HB 1 PM & 2,732 & 4,316 & 14,474 & 66,69 & 0,146 & 2,538 & 2,84 & 0 & 6,262 & 100 \\
\hline Différence & 1,42 & 1,5 & 1,63 & 9,49 & 0,73 & 0,67 & 1,2 & 0 & 8,31 & \\
\hline HB 2 PM & 0,326 & 1,96 & 13,004 & 75,18 & 0 & 1,84 & 3,512 & 0 & 4,176 & 100 \\
\hline Différence & 1,63 & 3,49 & 8,6 & 17,58 & 0 & 1,38 & 1,51 & 0 & 7,85 & \\
\hline HB 3 PM & 1,088 & 3,944 & 15,094 & 70,752 & 0 & 2,59 & 2,496 & 0 & 4,042 & 100 \\
\hline Différence & 2,35 & 1,68 & 3,33 & 7,66 & 0 & 0,72 & 3,48 & 0 & 5,57 & \\
\hline
\end{tabular}




\begin{tabular}{|c|c|c|c|c|c|c|c|c|c|c|}
\hline Échantillon & $\mathbf{N a}_{\mathbf{2}} \mathbf{O}$ & $\mathbf{M g O}$ & $\mathbf{A l}_{\mathbf{2}} \mathbf{O}_{\mathbf{3}}$ & $\mathbf{S i O}_{\mathbf{2}}$ & $\mathbf{P}_{\mathbf{2}} \mathbf{O}_{\mathbf{5}}$ & $\mathbf{K}_{\mathbf{2}} \mathbf{O}$ & $\mathbf{C a O}$ & $\mathbf{T i O}_{\mathbf{2}}$ & $\mathbf{F e}_{\mathbf{2}} \mathbf{O}_{\mathbf{3}}$ & Total \\
\hline HB 4 PM & 1,03 & 3,4 & 15,044 & 67,58 & 0,048 & 1,992 & 6,488 & 0,098 & 4,314 & 100 \\
\hline Différence & 3,46 & 1,49 & 7,98 & 18,15 & 0,24 & 1,62 & 10,36 & 0,49 & 5,6 & \\
\hline HB 5 PM & 0,902 & 3,448 & 17,97 & 67,782 & 0 & 2,108 & 1,962 & 0,218 & 5,606 & 100 \\
\hline Différence & 2,98 & 5,11 & 0,73 & 3,63 & 0 & 0,47 & 0,59 & 1,09 & 5,67 & \\
\hline HB 6 PM & 0,322 & 1,536 & 17,594 & 74,104 & 0 & 1,496 & 1,522 & 0 & 3,426 & 100 \\
\hline Différence & 1,61 & 3,93 & 3,36 & 18,68 & 0 & 2,58 & 2,67 & 0 & 6,83 & \\
\hline HB 7 PM & 0,216 & 3,012 & 13,45 & 73,652 & 0 & 2,564 & 2,75 & 0 & 4,354 & 100 \\
\hline Différence & 1,08 & 3,1 & 6,8 & 10,89 & 0 & 1,22 & 1,84 & 0 & 2,46 & \\
\hline HB 8 PM & 1,712 & 6,22 & 13,036 & 65,076 & 0 & 1,882 & 8,3 & 0 & 3,776 & 100 \\
\hline Différence & 4,69 & 4,22 & 3,86 & 20,07 & 0 & 1,02 & 13,08 & 0 & 6,39 & \\
\hline HB 9 PM & 0,774 & 3,962 & 16,696 & 68,57 & 0 & 2,246 & 3,568 & 0 & 4,184 & 100 \\
\hline Différence & 2,21 & 1,26 & 1,6 & 7,4 & 0 & 0,34 & 0,42 & 0 & 6,28 & \\
\hline HB 10 PM & 3,428 & 5,824 & 15,768 & 60,23 & 0,698 & 2,154 & 7,904 & 0 & 3,992 & 100 \\
\hline Différence & 7,86 & 4,9 & 2,49 & 3,39 & 1,4 & 1,73 & 5,56 & 0 & 3,95 & \\
\hline
\end{tabular}

Fig. 10 - Tableau de la composition élémentaire des matrices argileuses du groupe de San José de Moro (en \% d'oxydes; PM: moyenne; Ghavami 2016)

\begin{tabular}{|c|c|c|c|c|c|c|c|c|c|c|}
\hline Échantillon & $\mathbf{N a}_{\mathbf{2}} \mathbf{O}$ & $\mathbf{M g O}$ & $\mathbf{A l}_{\mathbf{2}} \mathbf{O}_{\mathbf{3}}$ & $\mathbf{S i O}_{\mathbf{2}}$ & $\mathbf{P}_{\mathbf{2}} \mathbf{O}_{\mathbf{5}}$ & $\mathbf{K}_{\mathbf{2}} \mathbf{O}$ & $\mathbf{C a O}$ & $\mathbf{T i O}_{2}$ & $\mathbf{F e}_{\mathbf{2}} \mathbf{O}_{\mathbf{3}}$ & Total \\
\hline SJM 1 PM & 0,458 & 3,544 & 15,558 & 59,746 & 0,49 & 1,834 & 14,528 & 0 & 3,838 & 100 \\
\hline Différence & 2,29 & 5,36 & 11,49 & 39,84 & 2,45 & 0,65 & 53,62 & 0 & 8,12 & \\
\hline SJM 2 PM & 2,834 & 4,256 & 14,736 & 63,492 & 0 & 2,226 & 7,436 & 0 & 5,022 & 100 \\
\hline Différence & 0,73 & 2,4 & 0,79 & 4,4 & 0 & 0,89 & 1,88 & 0 & 1,31 & \\
\hline SJM 3 PM & 0,746 & 4,23 & 13,854 & 62,848 & 0 & 2,16 & 11,288 & 0,348 & 4,53 & 100 \\
\hline Différence & 1,9 & 1,34 & 3,63 & 3,23 & 0 & 0,85 & 2,54 & 1,74 & 1,13 & \\
\hline SJM 4 PM & 3,72 & 4,92 & 16,334 & 60,822 & 0 & 2,576 & 6,736 & 0 & 4,892 & 100 \\
\hline Différence & 1,47 & 0,82 & 1,15 & 1,46 & 0 & 0,6 & 1,49 & 0 & 1,81 & \\
\hline SJM 5 PM & 0 & 3,34 & 14,848 & 60,382 & 0,448 & 2,48 & 12,818 & 0 & 5,684 & 100 \\
\hline Différence & 0 & 5,72 & 1,66 & 1,69 & 1,31 & 0,6 & 4,76 & 0 & 2,86 & \\
\hline SJM 6 PM & 1,432 & 4,288 & 16,182 & 63,284 & 0,596 & 2,196 & 8,782 & 0 & 3,24 & 100 \\
\hline Différence & 3,76 & 5,71 & 1,04 & 9,79 & 1,58 & 0,69 & 5,98 & 0 & 4,54 & \\
\hline SJM 7 PM & 1,998 & 3,756 & 15,18 & 61,042 & 0,816 & 2,45 & 10,584 & 0 & 4,168 & 100 \\
\hline Différence & 3,5 & 1,06 & 1,75 & 3,91 & 1,24 & 0,54 & 3,36 & 0 & 1,44 & \\
\hline SJM 8 PM & 2,058 & 3,584 & 14,806 & 66,152 & 0 & 1,892 & 6,496 & 0 & 5,012 & 100 \\
\hline Différence & 3,32 & 2,8 & 2,06 & 6,69 & 0 & 0,56 & 1,66 & 0 & 2,02 & \\
\hline SJM 9 PM & 1,748 & 3,59 & 16,594 & 61,588 & 0 & 3,14 & 6,944 & 0 & 6,394 & 100 \\
\hline Différence & 5,29 & 5,59 & 7,53 & 7,07 & 0 & 1,93 & 9,47 & 0 & 3,49 & \\
\hline SJM 10 PM & 2,108 & 3,776 & 14,516 & 56,848 & 0 & 3,014 & 10,528 & 0 & 9,206 & 100 \\
\hline Différence & 3,08 & 1,68 & 3,6 & 8,48 & 0 & 0,91 & 4,36 & 0 & 8,87 & \\
\hline
\end{tabular}


Entre Mochica et Lambayeque : retracer les identités de la période Transitionnelle

Fig. 11 - Tableau de la composition élémentaire des pâtes - comprenant la matrice argileuse et les inclusions du dégraissant - des céramiques de Huaca Bandera et de San José de Moro (en \% d'oxydes; Ghavami 2016)

\begin{tabular}{|c|c|c|c|c|c|c|c|c|c|}
\hline Échantillon & $\mathbf{N a}_{\mathbf{2}} \mathbf{O}$ & $\mathbf{M g O}$ & $\mathbf{A l}_{\mathbf{2}} \mathbf{O}_{\mathbf{3}}$ & $\mathbf{S i O}_{\mathbf{2}}$ & $\mathbf{P}_{\mathbf{2}} \mathbf{O}_{\mathbf{5}}$ & $\mathbf{K}_{\mathbf{2}} \mathbf{O}$ & $\mathbf{C a O}$ & $\mathbf{F e}_{\mathbf{2}} \mathbf{O}_{\mathbf{3}}$ & Total \\
\hline HB 1 & 0 & 4,01 & 13,15 & 66,76 & 0 & 2,44 & 8,39 & 5,26 & 100 \\
\hline HB 2 & 0 & 2,64 & 14,32 & 72,98 & 0 & 2,02 & 3,91 & 4,13 & 100 \\
\hline HB 3 & 2,73 & 3,43 & 13,29 & 70,82 & 0 & 2,07 & 3,01 & 4,66 & 100 \\
\hline HB 4 & 3,26 & 3,78 & 14,63 & 68,6 & 0,53 & 1,73 & 4,65 & 2,82 & 100 \\
\hline HB 5 & 2,54 & 4,09 & 16,23 & 68,73 & 0 & 2,57 & 1,61 & 4,24 & 100 \\
\hline HB 6 & 0 & 0 & 14,18 & 79,22 & 0 & 2,98 & 0 & 3,62 & 100 \\
\hline HB 7 & 0 & 3,3 & 14,66 & 71,59 & 0 & 2,66 & 3,1 & 4,69 & 100 \\
\hline HB 8 & 3,13 & 6,69 & 12,49 & 60,57 & 0 & 1,85 & 11,82 & 3,44 & 100 \\
\hline HB 9 & 2,1 & 4,13 & 16,5 & 66,7 & 0 & 2,21 & 3,75 & 4,61 & 100 \\
\hline HB 10 & 3,02 & 5,47 & 14,12 & 61,88 & 1,1 & 2,21 & 8,08 & 4 & 100 \\
\hline SJM 1 & 1,16 & 3,01 & 15,66 & 65,15 & 0,37 & 1,52 & 9,6 & 3,53 & 100 \\
\hline SJM 2 & 3,5 & 4,43 & 14,35 & 63,8 & 0 & 2,29 & 6,73 & 4,91 & 100 \\
\hline SJM 3 & 2,55 & 4,09 & 13,99 & 61,48 & 0,6 & 1,99 & 10,93 & 4,37 & 100 \\
\hline SJM 4 & 4,49 & 5,62 & 15,33 & 60,55 & 0,49 & 2,05 & 6,96 & 4,52 & 100 \\
\hline SJM 5 & 2,81 & 4,75 & 13,9 & 58,2 & 1,36 & 2,36 & 11,24 & 5,38 & 100 \\
\hline SJM 6 & 1,92 & 3,63 & 15,09 & 63,17 & 0,99 & 2,03 & 7,88 & 5,29 & 100 \\
\hline SJM 7 & 3,59 & 4,12 & 14,68 & 60,07 & 1,23 & 2,11 & 10,46 & 3,74 & 100 \\
\hline SJM 8 & 2,86 & 3,77 & 14,83 & 65,09 & 0 & 1,88 & 6,56 & 5,02 & 100 \\
\hline SJM 9 & 3,59 & 3,69 & 15,92 & 61,31 & 0 & 2,88 & 6,55 & 6,06 & 100 \\
\hline SJM 10 & 3,23 & 3,21 & 14,24 & 58,16 & 0 & 2,85 & 10,22 & 8,08 & 100 \\
\hline
\end{tabular}

En général, les résultats de l'analyse de la composition élémentaire des échantillons récupérés à Huaca Bandera et à San José de Moro permettent de différencier deux groupes de pâte chimiquement distincts (voir Figure 12). Les deux groupes étaient visiblement fabriqués avec des matières premières différentes, ce qui est attendu pour la production de céramiques utilitaires pour deux sites si distants. On observe principalement que l'argile de San José de Moro est plus homogène que celle de Huaca Bandera et se distingue de cette dernière par une teneur en silicium plus faible ainsi qu'une teneur en calcium plus élevée. Trois fragments de Huaca Bandera se démarquent néanmoins puisqu'ils montrent plus de similitudes avec le groupe de San José de Moro: HB 1, HB 8 et HB 10 (voir Figures 13 et 14). En effet, ces trois échantillons contiennent à la fois moins de silicium et plus de calcium que leur groupe. Ces teneurs sont particulièrement intéressantes puisque Rohfritsch (2006) a distingué deux types d'argiles dans la vallée de Jequetepeque: les calcaires ( $>6 \% \mathrm{Ca})$ et les non 
Fig. 12 - Diagramme ternaire présentant la teneur en silicium, aluminium, en magnésium, en calcium et en fer de la matrice argileuse des échantillons étudiés (en \% d'oxydes; Ghavami 2016)
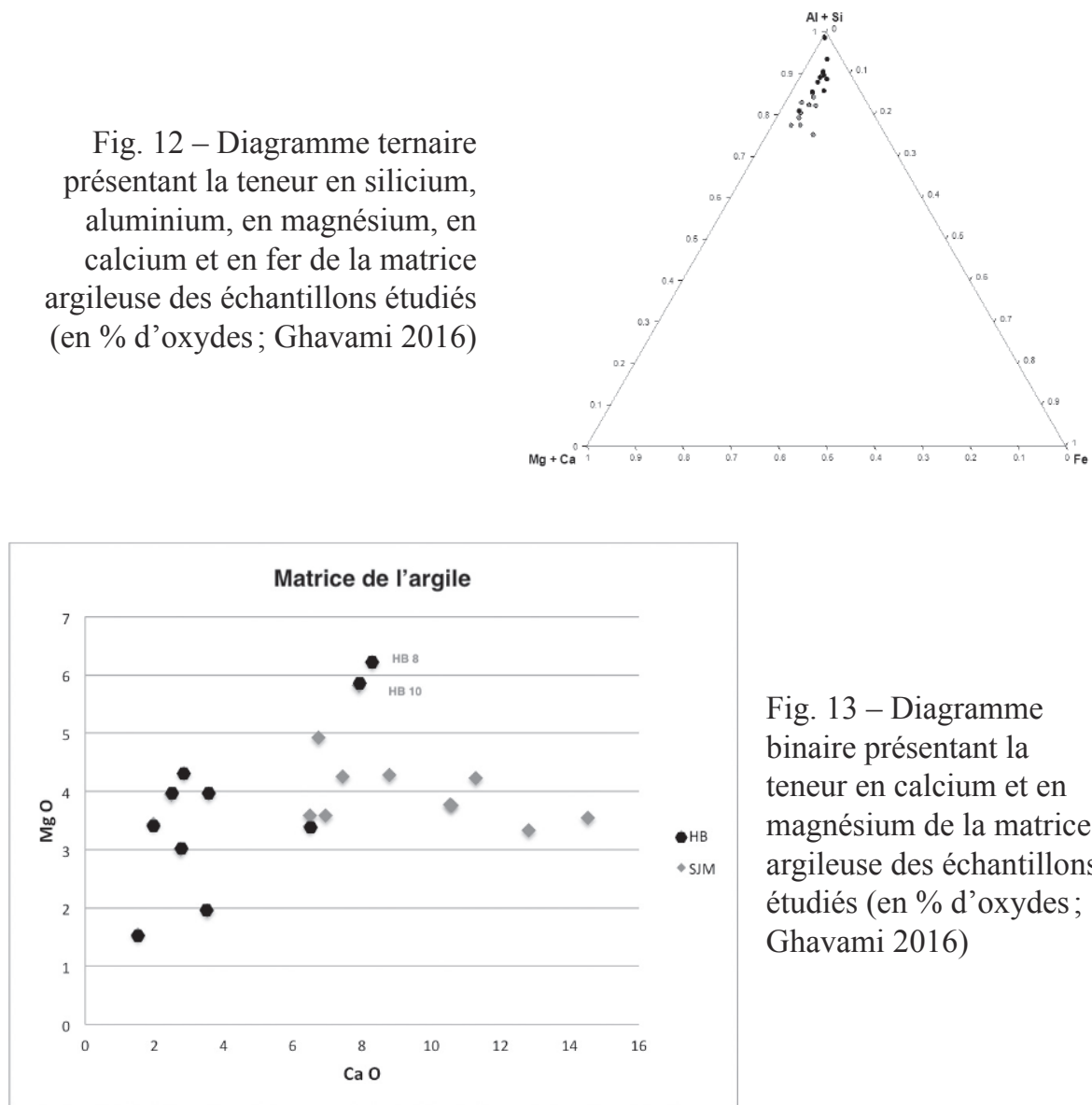

Fig. 13 - Diagramme binaire présentant la teneur en calcium et en magnésium de la matrice argileuse des échantillons étudiés (en \% d'oxydes; Ghavami 2016)

Fig. 14 - Diagramme binaire présentant la teneur en calcium et en Magnésium de la totalité d'une zone sélectionnée des échantillons étudiés (en \% d'oxydes; Ghavami 2016)

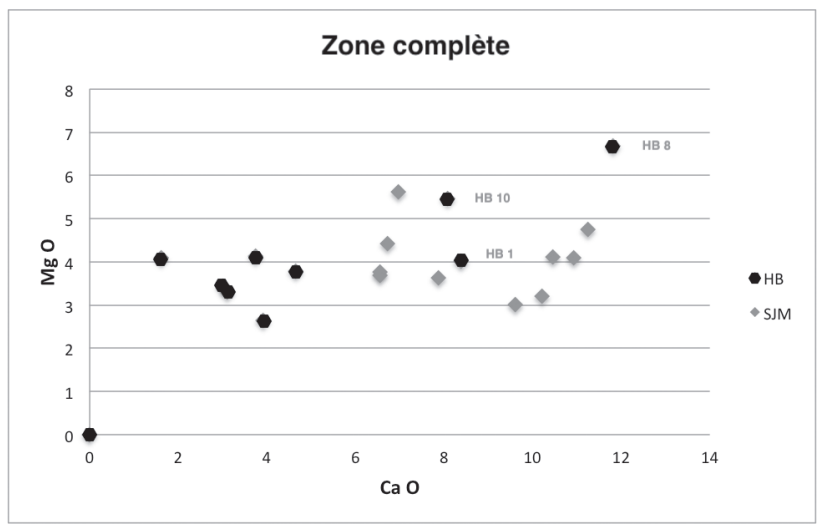


Entre Mochica et Lambayeque : retracer les identités de la période Transitionnelle

calcaires $(<6 \% \mathrm{Ca})$. Les résultats obtenus montrent que l'argile de San José de Moro appartient au premier groupe, tandis que celle de Huaca Bandera, de type siliceux, appartient au deuxième (voir Figures 15 et 16). Ces données pourraient laisser entendre que ces objets ont été transportés d'un site à l'autre pour une raison particulière.

Bien que les exemples d'importation de céramiques soient rares, l'analyse de la caractérisation des pâtes de la « ligne fine » du Mochica tardif (LFMT) de San José de Moro a montré qu'il existait visiblement deux productions différenciées pour ces objets prestigieux. Le premier groupe, plus abondant sur le site, se caractérise par des valeurs typiques de la vallée de Jequetepeque avec une argile calcaire, tandis que le second, moins représenté, est de type siliceux (Del Solar Velarde et al. 2016). Del Solar Velarde pense que le deuxième groupe de production de « ligne fine » pourrait provenir d'une autre vallée. Compte tenu des récentes découvertes de fragments de LFMT dans la vallée de Lambayeque,

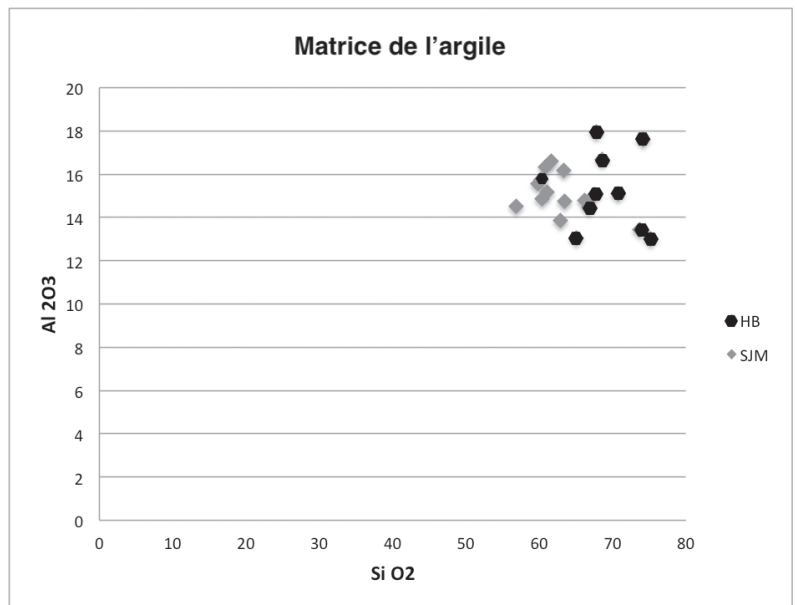

Fig. 15 - Diagramme binaire montrant la teneur en silicium et en aluminium de la matrice argileuse des échantillons étudiés (en \% d'oxydes; Ghavami 2016)

Fig. 16 - Diagramme binaire montrant la teneur en silicium et en aluminium de la totalité d'une zone choisie des échantillons étudiés (en \% d'oxydes; Ghavami 2016)

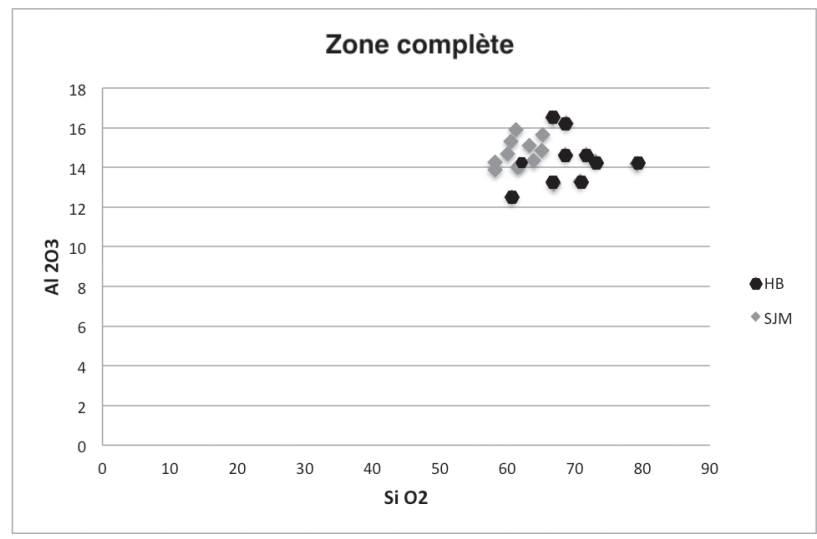


nous formulons l'hypothèse que ce deuxième groupe de production proviendrait des vallées de Lambayeque et de La Leche, attestant ainsi des relations étroites qu'entretenaient ces mêmes régions. En effet, ces différentiations de valeurs chimiques sont justement les mêmes que nous observons entre le groupe de production de San José de Moro et celui de Huaca Bandera (voir Figures 12 et 17) et pourrait trouver leur origine dans les caractéristiques géologiques des sédiments des vallées en question. De fait, l'agile utilisée pour la fabrication des céramiques étudiées provient, selon toute vraisemblance, des dépôts alluviaux Holocène qui dominent les parties avales des vallées de Jequetepeque et de Lambayeque. Ces dépôts sont formés par l'érosion de roches plus anciennes, de leur transport par les rivières et finalement de leur déposition. La composition de ces argiles est donc représentative de l'ensemble des roches présentes dans les bassins versants respectifs de chacune de ces vallées. Ces bassins comportent des roches très variées dominées par des sédiments siliciclastiques ou carbonatés du Paléozoïque et du Mésozoïque, des roches volcaniques du Cénozoïque et des roches plutoniques du Crétacé et du Cénozoïque (Ingemmet 2018). Ces types de roches présentent une minéralogie et une chimie extrêmement variées et se retrouvent dans chacun des deux bassins versants, rendant toute distinction basée sur la composition très hasardeuse. Toutefois, nous pourrions noter une légère différence dans les roches sédimentaires qui sont dominées par des âges Ordovicien à Jurassique formés de dépôts siliciclastiques dans le bassin de Lambayeque, alors que les sédiments du bassin de Jequetepeque sont dominés par des âges créactés qui contiennent plus de carbonates, ce qui pourrait expliquer les différenciations de valeurs chimiques que nous observons dans notre étude.

Une circulation d'objets dans la zone serait donc envisageable, même s'il serait peut-être plus concevable d'imaginer une telle situation pour le mobilier prestigieux, par exemple dans le cas où l'on viendrait déposer des offrandes lors d'une cérémonie funéraire. C'est pourquoi nous pouvons concevoir une autre explication qui aiderait à mieux comprendre ces occurrences en lien avec

Fig. 17 - Diagramme ternaire présentant la teneur en silicium, en aluminium, en Magnésium, en calcium et en fer de toute la zone choisie parmi les échantillons étudiés (en \% d'oxydes; Ghavami 2016)

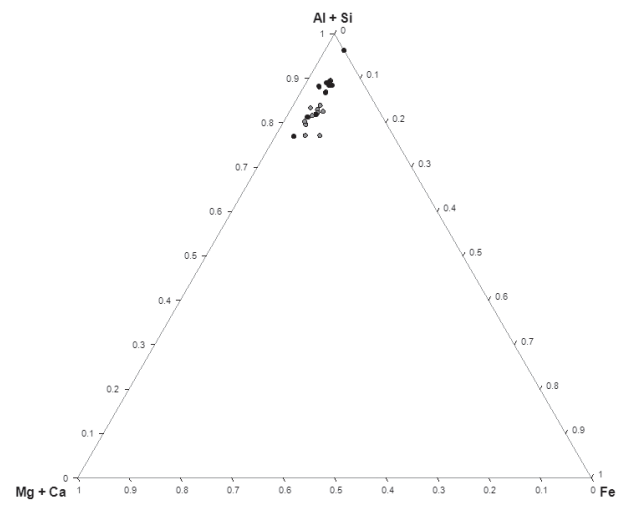


le matériel utilitaire. Les travaux ethnographiques ont attesté à de nombreuses reprises des cas de potiers itinérants dans les Andes, occasionnant un système de production particulier (Druc 2013; Ramón Joffré 2008, 2011, 2013). Ces derniers sont effectivement engagés dans la production de céramiques utilitaires durant une partie de l'année dans leur village et se déplacent le reste du temps dans différentes localités pour continuer leurs offices au gré des demandes. Il arrive que les artisans prennent leur propre matière première avec eux pendant les voyages ou alors qu'ils en trouvent sur place (Ramón Joffré 2013, p. 82). Ces cas nous avertissent de la nature potentiellement très dynamique des étapes de la chaîne opératoire, avant même la fabrication de l'objet. Ainsi, des communautés ordinairement éloignées qui ont recouru à des potiers itinérants pour subvenir à des besoins spécifiques auraient partagé un style de manufacture similaire. Ce système de productions particulier aurait-il pu s'appliquer à San José de Moro, où la consommation en céramique était considérable, comme le propose Gabriel Prieto (2008) pour l'époque Chimú? Et si c'était principalement les producteurs qui se déplaçaient?

\section{Discussion}

Les analyses archéométriques révèlent plusieurs aspects liés à la production de céramiques domestiques sur les sites de Huaca Bandera et de San José de Moro pour la fin de la période Mochica tardive et le début de la période Transitionnelle. Tout d'abord, il convient de souligner que, selon notre analyse typologique, les deux sites partagent les mêmes types morpho-fonctionnels. En revanche, il semble que chaque site fabriquait ses propres céramiques puisque l'analyse élémentaire démontre que la production n'était pas identique, malgré les grandes similitudes observées. La diversité de groupes minéralogiques démontre l'hétérogénéité des recettes de ce type d'artefacts qui semble avoir été produit dans une multitude d'ateliers. Selon Druc (2013), c'est une caractéristique attendue pour les objets utilitaires alors qu'à l'inverse, la production de biens prestigieux est généralement plus homogène et serait élaborée dans un nombre d'ateliers restreint. Selon l'auteur, « ce schéma de production différentielle n'exclut pas l'utilisation de ressources et de technologies locales, même si des céramiques spéciales ou fines ont été produites par des artisans recrutés, qu'ils soient locaux ou non. Cela n'exclut pas non plus la coexistence de différents types de production dans la même communauté » (ibid., p. 497). Ces modèles de production ont justement pu être attestés archéologiquement dans la fouille d'un atelier de Huaca del Sol y de la Luna (Chapdelaine, Kennedy et Uceda Castillo 1995), ou dans la vallée de Lurín où des techniques locales étaient employées pour produire différents styles, autant Inca impérial que local (Makowski et al. 2008). Le fait que la pâte des céramiques de Huaca Bandera semble plus hétérogène que celle de San José de Moro pourrait surprendre 
puisque le site se trouve relativement isolé. Cependant, cela pourrait justement s'expliquer par un recours à des potiers itinérants pour répondre adéquatement aux besoins du site, dont le ravitaillement permanent en matières premières de qualité s'avérait difficile en raison de son emplacement à l'orée du désert: une nécessité peut-être plus importante qu'à San José de Moro, où les dirigeants pouvaient bénéficier plus facilement de potiers spécialisés pour l'organisation des grandes cérémonies funéraires qui avaient lieu sur le site.

Quels mécanismes ont alors permis la grande similarité des types morphofonctionnels des céramiques domestiques entre les deux sites? Pour y répondre, il nous semble important de revenir sur la nature de la période de transition. Nous jugeons pertinent d'appuyer l'hypothèse de Rucabado-Yong (2006) selon laquelle l'effondrement de la culture mochica a été un phénomène progressif, de sorte que la sous-phase A était encore constituée d'une organisation essentiellement mochica. La sphère culturelle mochica résultant des interactions de plusieurs entités politiques autonomes était visiblement restreinte à seulement une poignée de communautés. Dans les lieux en question, comme San José de Moro et Huaca Bandera, les occupants auraient continué de conserver leurs liens ancestraux. Bien que des changements stylistiques aient été observés suite à l'influence huari durant l'Horizon moyen (ca. 550 apr. J.-C.-1000 apr. J.-C.), ils se sont produits de manière simultanée dans les deux sites alors qu'ils se trouvent à plus de $100 \mathrm{~km}$ de distance. D'ailleurs, l'abandon de Huaca Bandera coïncide avec le début de la sous-phase B de l'époque Transitionnelle à San José de Moro, caractérisée par une présence accrue d'éléments exogènes provenant de la région andine de Cajamarca. Existe-t-il un lien de cause à effet entre ces deux éventualités? L'hypothèse que nous soutenons est que les sites de San José de Moro et de Huaca Bandera appartenaient encore à une sphère d'interaction commune. La dynamique culturelle et les choix politiques de l'un influençaient le développement de l'autre, sans nécessairement que les contacts aient été directs, puisque nous avons trouvé des éléments architectoniques et des styles céramiques semblables à équidistance dans la vallée de Lambayeque (Bracamonte Lévano 2015). Les témoignages des relations économiques et culturelles de ces régions remontent d'ailleurs à des périodes encore plus anciennes. De fait, les expressions de la poterie et de la métallurgie se sont développées de manière analogue depuis la période Mochica moyenne. Des études sur les toponymes des vallées de Jequetepeque, de Lambayeque et de La Leche indiquent que les habitants de cette aire géographique partageaient la même langue, le muchik, et que la construction de canaux d'irrigation intervallées permettait de relier ces régions dans un même système hydraulique (voir Cerrón-Palomino 1995, Kosok 1965). Ces multiples éléments attestent d'une trajectoire culturelle commune à ces territoires, qui auraient pourvu un contexte favorable aux échanges de produits spécifiques et à la circulation de biens divers et de personnes. 
Entre Mochica et Lambayeque : retracer les identités de la période Transitionnelle

Relevons en outre que, malgré les efforts déployés pendant les fouilles, aucune sépulture correspondant à la période d'occupation n'a encore été mise à jour sur le site de Huaca Bandera. La monumentalité du site nous indique qu'il serait très probable que l'élite ait pris soin d'enterrer les siens dans de somptueux tombeaux. Dans ce cas, où l'ont-ils fait? Compte tenu de la fonction de Huaca Bandera - un site combinant des aspects à la fois administratifs et cérémoniels - et de celle de San José de Moro - un centre cérémoniel hébergeant un cimetière d'élite où d'importants rituels étaient célébrés autour du culte des ancêtres -, nous pouvons faire l'hypothèse que leur nature différenciée, mais complémentaire, a pu être l'une des raisons principales de leurs interactions. De plus, San José de Moro était un lieu de culte dont l'occupation saisonnière dépendait des communautés environnantes. Ne peut-on pas penser qu'un site comme San José de Moro ait pu servir de lieu d'inhumation pour l'élite mochica, au-delà même de la vallée de Jequetepeque? En effet, le centre cérémoniel jouissait d'une grande réputation pour tout individu de cette tradition, puisqu'il hébergeait les huacas d'une série d'illustres ancêtres directement liés à la narration mythique mochica. Par conséquent, nous pensons que les relations entre Huaca Bandera et San José de Moro ont pu être stimulées par la complémentarité qui caractérise la fonction des deux sites. Selon Renfrew (1986), le système d'interaction entre « entités politiques de pairs » influence le développement sociopolitique des groupes affiliés, créant ainsi des similitudes dans leur production matérielle, leur idéologie et leur structure organisationnelle de manière relativement contemporaine. Il ne serait alors pas inapproprié d'imaginer qu'en période de crise et de perte de légitimité idéologique, l'élite mochica de Huaca Bandera ait décidé d'entreprendre le pèlerinage à San José de Moro. Cela aurait pu lui permettre de se rapprocher symboliquement d'un prestigieux axis mundi, de sorte à revendiquer son identité face aux nouvelles traditions venues des Andes tout en renforçant ses liens ancestraux dans un lieu sacré. En ce sens, cette stratégie aurait conjointement inclus les deux sites dans le même réseau d'interaction mochica. On a par ailleurs retrouvé à Huaca Bandera les mêmes structures architecturales de type plateforme exhumées par Swenson (2004) dans la vallée de Jequetepeque et que Rucabado-Yong (2006) a reconnues dans la configuration de la chambre funéraire M-U615 à San José de Moro.

Ce réseau d'interactions, auquel auraient participé les communautés de San José de Moro et de Huaca Bandera au début de la période Transitionnelle, refléterait toutefois une stratégie d'ordre politique plutôt qu'un lien ethnique. Cela impliquerait que, moyennant l'obtention et l'utilisation d'un attirail religieux caractéristique, les communautés exprimaient l'intention de s'incorporer à la sphère politique mochica. Cet élément nous renvoie à la question de la conscience du groupe dans l'affirmation de son identité lors des interactions sociales. Étant donné que le matériel utilitaire que nous avons analysé ne traduit pas l'aspect communicatif de l'objet mais plutôt sa fonction, les éléments qui le 
caractérisent ne sont pas de nature à revendiquer intentionnellement une identité particulière (Figure 18) ${ }^{3}$. De ce fait, ce réseau d'interactions n'explique pas à lui seul les similarités au niveau morpho-fonctionnel des céramiques utilitaires que nous avons analysées. Ceci nous suggère qu'un autre système socio-économique en plus du réseau d'interactions « politico-religieux » mochica a occasionné les synergies culturelles que nous observons.

Fig. 18 - Tableau récapitulatif des caractéristiques générales des types de céramiques et des analyses correspondantes réalisées dans ce travail (Ghavami 2016)

\begin{tabular}{|c|c|c|}
\hline & Groupe 1 & Groupe 2 \\
\hline Objet & Céramique fine, « prestigieuse » & Céramique utilitaire, domestique \\
\hline But & Communication & Fonction \\
\hline Caractéristique & $\begin{array}{c}\text { «Symbole culturel » } \\
\text { Intentionnel - revendiqué - actif }\end{array}$ & $\begin{array}{l}\text { «Empreinte ethnique » } \\
\text { Non intentionnel - passif }\end{array}$ \\
\hline Production & Contrôlée, homogène & Libre, hétérogène \\
\hline Changement & Dynamique & Stable - constant \\
\hline $\begin{array}{l}\text { Courant } \\
\text { théorique }\end{array}$ & Interactions sociales (Barth) & $\begin{array}{l}\text { Techniques du corps (Mauss) } \\
\text { Pratique (Bourdieu) }\end{array}$ \\
\hline Observation & $\begin{array}{c}\text { «Style décoratif } » \\
\text { (Décoration }- \text { forme - fonction) }\end{array}$ & $\begin{array}{c}\text { « Style technique » } \\
\text { (Forme - fonction - production) }\end{array}$ \\
\hline Analyse & $\begin{array}{l}\text { Observation typologique, accent } \\
\text { sur les éléments décoratifs } \\
\text { - niveau macroscopique }\end{array}$ & $\begin{array}{l}\text { Élémentaire, chimique - niveau } \\
\text { microscopique }\end{array}$ \\
\hline Identité(s)/Lien & $\begin{array}{l}\text { Politique - économique } \\
\text { - religieux }\end{array}$ & Ethnique \\
\hline Communauté & Niveau régional & Niveau local \\
\hline $\begin{array}{c}\text { Sphère } \\
\text { d'interactions }\end{array}$ & Culture mochica & $\begin{array}{l}\text { Interactions des potiers } \\
\text { (itinérants ou non) }\end{array}$ \\
\hline
\end{tabular}

Au cours de ses recherches ethnographiques sur le terrain, Gabriel Ramón Joffré a observé des cas où les carrières étaient partagées par plusieurs communautés de potiers qui, en fréquentant le même lieu et en utilisant les mêmes matières premières, créaient une sphère $d$ 'interaction leur permettant de partager et de transmettre des informations générales sur les techniques de fabrication et l'utilisation de l'argile (Ramón Joffré 2008, 2013). Ces pratiques seraient à l'origine de la répartition géographique et de la conception d'un même style

3. Ce tableau propose une division schématisée et logique dans le but d'encourager la réflexion sur le lien entre le style matériel et l'identité. Les corrélations présentées ne devraient pas être définitives puisque les catégories peuvent s'entrecroiser. En effet, il est aussi encouragé de procéder à des analyses élémentaires sur le mobilier prestigieux. 
technique commun à plusieurs communautés éloignées, ce qui pourrait expliquer les similitudes morpho-fonctionnelles de la céramique utilitaire des sites que nous étudions. « Les pratiques et les connaissances liées au traitement des matières premières, leur nomenclature et les rituels en relation avec les sources constituent une base culturelle commune entre ces communautés » (Ramón Joffré 2013, p. 69). Ce modèle pourrait d'ailleurs s'appliquer au niveau régional si l'on considère la superposition d'aires de distribution qui permettrait aux communautés éloignées de partager indirectement plusieurs aspects de leur production, notamment grâce aux échanges favorisés par la mobilité de potiers itinérants et à l'interaction de voisins en commun. Les potiers itinérants auraient eu la possibilité d'exercer leurs offices dans une aire culturelle préexistante, liée à la sphère d'interactions mochica, qui aurait intégré les vallées de Lambayeque, de La Leche et de Jequetepeque dans un même système. Seulement, à l'inverse de cette dernière, le deuxième modèle n'opère pas de manière stratégique mais se développe plutôt spontanément dans le substrat socio-économique. Cette distinction rappelle dans une certaine mesure la différence fondamentale que Claude Lévi-Strauss a définie entre l'histoire et l'ethnologie. Selon lui, la première discipline organise ses données par rapport aux expressions conscientes, alors qu'à l'inverse la seconde le fait par rapport aux conditions inconscientes, de la vie sociale (Lévi-Strauss 2003 [1958], p. 31). En ce sens, si les communautés mochica partageant le même style justifiaient par l'usage de symboles et d'images répétés une mémoire collective et un ordre établi, les techniques employées à ces fins sont le fruit de phénomènes complexes et variés combinant l'ensemble de savoir-faire et de pratiques perçus par la société comme d'anciennes traditions héritées et donc non justifiées.

\section{Conclusion}

En vue d'interpréter les résultats de notre analyse à la lumière de cette discussion, nous proposons un modèle hypothétique pour la production de céramiques et ses implications pour la fin de la période Mochica et le début de la phase Transitionnelle sur la côte nord du Pérou. Les cas signalés nous permettent de constater toute une gamme de productions et de diffusions de styles décoratifs et techniques qui peuvent expliquer les importantes similitudes entre les types morpho-fonctionnels des céramiques domestiques récupérés sur les sites de Huaca Bandera et de San José de Moro. Nous estimons qu'il existait au moins deux sphères d'interactions propres traduisant différentes dimensions des identités collectives. La première, liée à la sphère politico-religieuse mochica, était articulée par des cérémonies faisant allusion au culte des ancêtres mythiques et aurait influé davantage sur la production d'objets prestigieux principalement sous leurs aspects décoratifs et symboliques. La seconde concernait la circulation de potiers - itinérants ou non - et la transmission de leurs connaissances 
par le biais de carrières communes et de procédés particuliers. Celle-ci aurait fonctionné indépendamment ou en fonction des besoins et des demandes pour la production de biens prestigieux. C'est la sphère qui aurait le plus influencé le style technique des potiers, en reflétant les aspects traditionnels de production.

Dans cette optique, nous pourrions compléter la proposition de Makowski qui distinguait deux types d'identités liées aux styles culturels des sociétés andines. Si, selon lui, le style emblématique de la culture mochica reflète dans son ensemble l'identité politique de l'utilisateur, l'identité ethnique n'est, quant à elle, associée qu'aux styles culturels des sociétés moins complexes comme la culture virú-gallinazo (Makowski 2010). Pour notre part, nous considérons que le mobilier utilitaire peut aussi traduire des aspects de l'identité ethnique du producteur ou de l'utilisateur, étant donné que ce matériel culturel reflète intrinsèquement les processus de production traditionnels ainsi que les interactions spontanées, même au sein des sociétés complexes.

Toutefois, les changements produits durant l'Horizon moyen ont eu une incidence sur la production d'artefacts mochica, puisque la sphère d'interaction des potiers a, elle aussi, été influencée par les mouvements de personnes apportant de nouvelles idées. Les éléments atypiques introduits dans la production de céramiques ont transformé le paysage en évoquant un univers différent en contradiction avec le vieux monde mochica. Ce revirement idéologique, associé à des développements socio-économiques complexes, a redéfini le contexte socioculturel de la côte nord, y compris au niveau ethnique. *

* Manuscrit reçu en mai 2020, accepté pour publication en avril 2021.

\section{Références citées}

BARTH Fredrik G.

1969 Ethnic Groups and Boundaries. The Social Organization of Culture Difference, Little, Brown and Company, Boston.

Bourdieu Pierre

1972 Esquisse d'une théorie de la pratique, précédé de Trois études d'ethnologie Kabyle, Librairie Droz, Genève.

BraCAMONTE LÉvANo Edgar

2015 Huaca Santa Rosa de Pucalá y la organización territorial del valle de Lambayeque, Ministerio de Cultura del Perú, Unidad Ejecutora 005, Proyecto Especial Naylamp Lambayeque, Lambayeque.

2019a «Un recinto Wari con forma "D" », in La Industria, Suplemento Dominical, édition du 13 janvier 2019, Chiclayo, p. 4-5.

2019 b « La cerámica cajamarca como evidencia de interacción social en Santa Rosa de Pucalá, valle medio de Lambayeque, durante el Horizonte Medio », in 
Entre Mochica et Lambayeque : retracer les identités de la période Transitionnelle

Actas del V Congreso Nacional de Arqueología, Ministerio de Cultura del Perú, Lima, vol. 1, p. 25-41.

Cabello de Balboa Miguel

1951 [1586] Miscelánia Antártica. Una historia del Perú Antiguo, Universidad Nacional Mayor de San Marcos, Lima.

CAstillo Luis Jaime

2001 «The last of the Mochicas, a view from de Jequetepeque Valley », in Joanne Pillsbury (dir.), Moche Art and Archaeology in Ancient Peru, Center for the Advanced Study of the Visual Arts, National Gallery of Art (Studies in the History of Art, 63), Washington (DC), p. 307-332.

2012 San José de Moro y el Fin de los Mochicas en el Valle de Jequetepeque, Costa Norte del Perú, thèse de doctorat en anthropologie, Faculté des études supérieures, University of California, Los Angeles.

Cerrón-Palomino Rodolfo

1995 La lengua de Naimlap. Reconstrucción y obsolescencia del mochica, Fondo Editorial PUCP, Lima.

Chapdelaine Claude, Greg Kennedy et Santiago Uceda Castillo

1995 «Activación neutrónica en el estudio de la producción local de la cerámica ritual en el sitio Moche, Perú », Bulletin de l'Institut français d'études andines, 24 (2), p. 183-212.

Childe V. Gordon

1929 The Danube in Prehistory, Clarendon Press, Oxford (UK).

Curo Chambergo Manuel

2013 Informe final Temporada 2013, Proyecto de Investigación Arqueológica Huaca Bandera, Ministerio de Cultura del Perú, Unidad Ejecutora 005 MC, Naylamp/Lambayeque, Museo Arqueológico Nacional Brüning, Lambayeque.

CURTA Florin

2014 «Ethnic identity and archaeology », in Claire Smith (dir.), Encyclopedia of Global Archaeology, Springer, New York.

Cusicanqui Marsano Solsiré

2010 Investigaciones arqueológicas en los asentamientos de San Ildefonso y Cerro Chepén, valle bajo de Jequetepeque, thèse de licence en archéologie, Pontificia Universidad Católica del Perú, Lima.

Del Solar Velarde Nino, Stan Kinis, Rémy Chapoulie, Luis Jaime Castillo et Renaud JOANNES-BOYAU

2016 «Characterization of pre-Columbian artefacts in situ through handheld portable X-ray fluorescence spectrometry: the case of ceramics from the Mochica site of San José de Moro (Peru) », Heritage Science, 4, Article number: 37, https:// doi.org/10.1186/s40494-016-0109-y.

DiAz-AndReu Margarita et Sam LuCY

2005 The Archaeology of Identity. Approaches to gender, age, status, ethnicity and religion, Routledge, New York/London.

Donnan Christopher B.

1990 " An assessment of the validity of the Naylamp Dynasty », in Michael Moseley et Alana Cordy-Collins (dir.), The Northern Dynasties. Kinship and Statecraft 
in Chimor. A Symposium at Dumbarton Oaks, Dumbarton Oaks Research Library and Collection, Washington (DC)

Donnan Christopher B. et Carol Mackey

1978 Ancient Burial Pattern of the Moche Valley, Perú, University of Texas Press, Austin.

Donnan Christopher B. et Donna McClelland

1999 Moche Fineline Painting. Its Evolution and its Artists, Fowler Museum of Cultural History, University of California, Los Angeles.

DRUC Isabelle

1996 Caractérisation et analyse de provenance de la production céramique dans l'aire d'influence Chavín (Andes centre-nord), un aspect des relations interrégionales à l'Horizon ancien, thèse de doctorat en anthropologie, Faculté des études supérieures, Université de Montréal, Montréal.

2013 «What is local? Looking at ceramic production in the Peruvian Highlands and beyond », Journal of Anthropological Research, 69, p. 485-513.

GAUTHIER François

2019 Religion, Modernity, Globalisation. Nation-State to Market, Routledge (Studies in Religion), London.

GeERTz Clifford

1973 The Interpretation of Cultures, Basic Books, New York.

GHavami Sâm

2016 Rastreando identidades Post Mochica, thèse de Magister en archéologie avec mention d'études andines, Pontificia Universidad Católica del Perú, Lima.

Gosselain Olivier P.

2000 «Materializing identities: an African perspective », Journal of Archaeological Method and Theory, 7 (3), p. 187-217.

HodDER Ian

1982 Symbols in action. Ethnoarchaeological studies of material culture, Cambridge University Press, Cambridge et New York.

InGEMmet (Instituto Geológico Minero y Metalúrgico)

2018 Mapa geológico del Perú 1:1 000 000, https://portal.ingemmet.gob.pe/web/ guest/mapa-geologico-nacional, consultée le 01/07/2021.

JONES Siân

1996 The Archaeology of Ethnicity. Constructing identities in the past and present, Routledge, New York/London.

Kossinna Gustaf

1911 Die Herkunft der Germanen, Kabitzsch, Leipzig.

Kosok Paul

1965 Life, Land and Water in Ancient Peru, Long Island University, New York.

LARCO Rafael

1948 Cronología Arqueológica del Norte del Perú, Biblioteca del Museo de Arqueología Rafael Larco Herrera, Sociedad Geográfica Americana, Buenos Aires.

Lavalle Jose Antonio

1989 Culturas Precolombinas: Lambayeque, Banco de Crédito del Perú (Arte y Tesoros del Perú), Lima. 
Entre Mochica et Lambayeque : retracer les identités de la période Transitionnelle

LÉvi-STRAuss Claude

2003 [1958] Anthropologie structurale, Plon (Pocket), Paris.

LuCY Sam

2005 «Ethnic and cultural identities », in Margarita Diaz-Andreu et Sam Lucy (dir.), Archaeology of Identity, Routledge, New York/London, p. 86-109.

MaKowski Krzysztof

2010 «Religion, ethnic identity, and power in the Moche world: a view from the frontiers », in Jeffrey Quilter et Luis Jaime Castillo (dir.), New Perspectives on Moche Political Organization, Dumbarton Oaks Research Library and Collection (Dumbarton Oaks Pre-Columbian Symposia and Colloquia), Washington (DC), p. 278-303.

Makowski Krzysztof, Ivan Ghezzi, Daniel Guerrero, Hector NefF, Milagritos JimÉnez, Gabriela Oré Menendez et Rosabella Álvarez

2008 «Pachacamac, Ychsma y los Caringas: estilos e identidades en el valle de Lurín Inca », in Omar Pinero et Henri Tantaleán (dir.), Arqueología de la Costa Centro Sur Peruana, Avqi Ediciones, Lima, p. 267-316.

Mauss Marcel

1934 «Les techniques du corps », Journal de Psychologie, 32 (3-4), communication présentée à la Société de Psychologie le 17 mai 1934.

McClelland Donna

1990 « A maritime passage from Moche to Chimu », in Michael Moseley et Alana Cordy-Collins (dir.), The Northern Dynasties. Kinship and Statecraft in Chimor. A Symposium at Dumbarton Oaks, Dumbarton Oaks Research Library and Collection, Washington (DC), p. 75-106.

MuRO-YNOÑAn Luis

2009 Espacios públicos, encuentros sociales y ritual funerario en San José de Moro. Análisis de la ocupación Mochica Tardio en el Área 45, Sector Oeste de San José de Moro, thèse de licence en archéologie, Pontificia Universidad Católica del Perú, Lima.

OrÉ Menendez Gabriela

2012 Los alfareros del valle de Pachacámac: relaciones costeño-serranas a través del análisis arqueométrico de la cerámica, thèse de Magíster en archéologie avec mention d'Études andines, Pontificia Universidad Católica del Perú, Lima.

PriETo Gabriel

2008 «Cerámica utilitaria Chimú de San José de Moro: Tipología de formas y modelos interpretativos », Revista del Museo de Arqueología, Antropología e Historia, 10, p. 111-154.

RAMÓN JOFFRÉ Gabriel

2008 «Producción alfarera en Piura (Perú): estilos técnicos y diacronía », Bulletin de l'Institut français d'études andines, 37 (3), p. 477-509.

2011 "The swallow potters: seasonally migratory styles in the Andes », in Simona Scarcella (dir.), Archaeological Ceramics. A Review of Current Research, Archaeopress, Oxford, p. 160-175. 
RAMÓN JOFFRÉ Gabriel

2013 «Las fuentes del estilo: distribución regional de canteras y técnicas alfareras en Conchucos (Ancash, Perú) », Bulletin de l'Institut français d'études andines, 42 (1), p. 49-90.

RENFRew Colin

1986 «Introduction: peer polity interaction and socio-political change », in Colin Renfrew et John F. Cherry (dir.), Peer Polity Interaction and Socio-Political Change, Cambridge University Press, Cambridge, p. 1-18.

RoHFritsch Agnès

2006 Céramiques mochicas de la vallée de Jequetepeque (Pérou) : étude technique et physico-chimique d'exemplaires provenant de Dos Cabezas et San José de Moro, travail de mémoire pour l'obtention du titre de Magister en archéo-

Rosas Marco logie, Université Michel de Montaigne, Bordeaux 3.

2007 « Nuevas perspectivas acerca del colapso Moche en el bajo Jequetepeque: Resultados preliminarios de la segunda campaña de investigación del proyecto arqueológico Cerro Chepén », Bulletin de l'Institut français d'études andines, 36 (2), p. 221-240.

Rowe John

1948 «The Kingdom of Chimor », Acta Americana, 6 (1-2), Mexico, p. 26-59.

RuCABADO-Yong Julio

2006 Elite Mortuary Practices at San José de Moro during the Transitional Period. The Case Study of Collective Burial M-U615, thèse de Magister en archéologie, University of North Carolina, Chapel Hill.

2008 «Prácticas funerarias de elite en San José de Moro durante la fase Transicional Temprana », in Luis Jaime Castillo, Hélène Bernier, Gregory Lockard et Julio Rucabado-Yong (dir.), Arqueología mochica. Nuevos enfoques, actes du Primer Congreso Internacional de Jóvenes Investigadores de la cultura mochica (4 y 5 de agosto de 2004), Fondo Editorial de la Pontificia Universidad Católica del Perú, Lima, p. 359-380.

RucABAdo-Yong Julio et Luis Jaime Castillo

2003 «El Periodo Transicional en San José de Moro », in Santiago Uceda et Elías Mujica (dir.), Moche Hacia el Final del Milenio. Actas del Segundo Coloquio sobre la Cultura Moche, Universidad Nacional de Trujillo y Pontificia Universidad Católica del Perú, Lima, vol. 1, p. 15-42.

SHIMADA Izumi

1981 «The Batan Grande - La Leche Archaeological Project: the first two seasons », Journal of Field Archaeology, 8 (4-Winter), p. 405-446.

1985 «La cultura Sicán: caracterización arqueológica », in Eric Mandoza Samillán (dir.), Presencia histórica de Lambayeque, Ediciones y Representaciones H. Falconí, [Peru], p. 76-131.

SwENSON Edward

2004 Ritual and power in the urban hinterland: religious pluralism and political decentralization in Late Moche Jequetepeque, Peru, thèse de doctorat en anthropologie, Faculté des études supérieures, Université de Chicago, Chicago. 